\title{
A Joint Inversion-Segmentation approach to Assisted
}

\section{Seismic Interpretation}

\author{
Matteo Ravasi ${ }^{1}$, Claire Birnie ${ }^{1}$ \\ ${ }^{1}$ KAUST, Thuwal, 23955, Kingdom of Saudi Arabia
}

\section{SUMMARY}

Structural seismic interpretation and quantitative characterization are historically intertwined processes. The latter provides estimates of the properties of the subsurface, which can be used to aid structural interpretation alongside the original seísmic data and a number of other seismic attributes. In this work, we redefine this process as an inverse problem which tries to jointly estimate subsurface properties (i.e., acoustic impedance) and a piece-wise segmented representation of the subsurface based on user-defined macroclasses. By inverting for the quantities simultaneously, the inversion is primed with prior knowledge about the regions of interest, whilst at the same time it constrains this belief with the actual seismic measurements. As the proposed functional is separable in the two quantities, these are optimized in an alternating fashion, where each subproblem is solved using the Primal-Dual algorithm. Subsequently, the final segmented model is used as input to an ad-hoc workflow that extracts the perimeter of the detected shapes and produces a structural framework (i.e., seismic horizons) consistent with the estimated subsurface properties. The effectiveness of the proposed method is illustrated through numerical examples on synthetic and field datasets.

Key words: Inverse theory - Numerical solutions - Computational seismology - Image processing. 


\section{INTRODUCTION}

Seismic interpretation forms part of an integrated workflow for mapping and characterization of subsurface deposits (Brown 2011; Herron 2011), with applications ranging from hydrocarbon (Ashcroft 2011) and geothermal exploration (Majer 2003; Bredesen et al. 2021) all the way to monitoring of carbon capture and storage sites (Mulrooney et al. 2018; Angelo et al. 2019). It is traditionally divided into two components: structural and quantitative interpretation. The former aims at identifying the geological framework (i.e., horizons and faults) whilst the latter focuses on retrieving properties of the subsurface. Structural interpretation is nowadays mostly carried out manually and led by geological and geophysical understanding of the subsurface. More specifically, key geophysical principles are followed to identify an interpretation strategy that guides the interpreter's job: with the aid of sonic and density logs, a first step in any interpretation project is represented by the creation of well-ties, which allow interpreters to link the geological stratigraphy to the observed seismic response. In other words, interpreters perform seismic modelling to identify changes in elastic properties that trigger a seismic response in the observed data. However, as the word implies, interpretation is a subjective matter whose ultimate goal is that of reconstructing the geological story contained within a seismic volume (Herron 2011).

With recent advances in artificial intelligence and deep learning (DL), several attempts at automatic seismic interpretation have been reported (Waldeland et al. (2018); Haibin et al. (2019); Shi et al. (2019); Shi et al. (2020)). A common feature of most of these approaches is that of turning a geologically-driven process into a computer vision and pattern recognition task. Such DL-based methods have been shown to be very successful for some specific tasks where a clear pattern can be observed in the data and learned - e.g., top salt interpretation (Waldeland et al. 2018), where a seismically rich area is abruptly interrupted by a strong reflector, followed by an area with little to no seismic signal. On the other hand, these methods currently fail at integrating multiple data types in the learning process, something that human interpreters are very good at. For example, since well-tie principles cannot be directly encoded in the machine learning training process, interpreters are still required to define the horizons of interest and interpret them for a number of lines before training a machine to subsequently auto-track these horizons of interest in the rest of the dataset (Haibin et al. 2019).

Post-stack inversion (Sheriff 2002) and pre-stack inversion (Brown 2011; Hampson et al. 2005) represent the workhorses for quantitative characterization of the subsurface. Post-stack inversion has been originally developed as a single trace method based on the assumption that seismic traces can be modelled using the convolutional and vertical incidence reflection coefficient equation (Russell \& Hampson 2011). Similarly, pre-stack or amplitude-versus-offset (AVO) inversion relies on a trace- 
by-trace modelling of pre-stack seismic data based on angle dependent reflectivity modelling via a linearisation of the Zoeppritz equation (Simmons \& Backus 1996). Several extensions of the original methods have been proposed in the literature to include additional physical constraints aimed at enforcing correlation between elastic parameters in either a deterministic (Hampson et al. 2005) or Bayesian (Buland \& Omre 2003) fashion. Similarly, joint inversion of multiple datasets (e.g., PP and PS AVO gathers - Margrave et al. (2001)) is also used as a way to further constrain the problem. However, since the input seismic data are band-limited in nature and lack both the very low and high frequencies, traditional inversion approaches are unable to completely recover the full-spectrum reflectivity, leading to unwanted oscillatory behaviors near interfaces in the recovered model. While a properly defined low frequency background model can partially fill such a frequency gap, the remaining missing frequencies can only be compensated for by providing additional prior information in the form of suitable regularizers. To this extent, the fundamental differences between different algorithms lies in the choíce of the regularizers and how the missing low frequencies are injected into the final solution. For example, the widely used sparse-spike inversion (Oldenburg et al. 1983) tries to enforce blockiness in the inverted model for both post- and pre-stack inversion by first inverting for a sparse reflectivity of the Earth, which is subsequently integrated over the time (or depth) axis to retrieve a blocky model of the elastic properties in the subsurface. Alternatively, model-based methods such as Cooke \& Schneider (1983) start from an initial model of the subsurface properties and utilize seismic data to refine such a model by means of a least-squares functional with the possible addition of spatial regularization terms. Total-Variation (TV) regularization is another form of regularization that can promote blockiness in the recovered subsurface model; using this regularization does however come at the cost of making the overall functional non-smooth, something that cannot be easily minimised using popular least-squares direct or iterative solvers (Parikh 2013). Luckily, this family of non-smooth, convex functionals have been extensively studied in other fields of science, and so-called proximal solvers have been proposed to efficiently optimize such functionals. Within the geophysical community, early adoption of one such solver called the Alternating Direction Method of Multipliers (ADMM) has been suggested by Wang et al. (2019) and Kolbjornsen et al. (2019) for both post- and pre-stack inversion of band-limited data. TV regularization is however known to lead to a systematic loss of contrast (Benning \& Burger 2013), which when used for seismic inversion may result in underprediction of subsurface properties. A more advanced solver that overcomes this limitation is the Split-Bregman algorithm of Goldstein \& Osher (2009); such a solver has been successfully employed in solving L1-regularized problems in a variety of fields including geophysics: it is for example used by Gholami (2015) in the context of seismic impedance inversion of reflectivity data (after a preliminary step of blind deconvolution) and by Qiu et al. (2016) for full-waveform inversion. 
Finally, since geophysical inversion products are generally used as part of the interpretation process that seeks to differentiate geologic units, several authors have recently suggested the use of a clustering constraint that pushes the inversion process to select models primarily composed of a number of expected discrete rock units. The fuzzy c-means algorithm has been used in the context of seismic tomography (Sun \& Li 2015), gravity inversion (Sun et al. 2001), and seismic inversion (Kieu $\&$ Kepic 2020). Whilst such constraints have shown great promise and helped the solution to be more consistent with geological prior knowledge of the subsurface, the clustering algorithm has a local effect and cannot be informed with spatial information of nearby subsurface locations. An alternative way to include such a constraint is to recast it mathematically as a segmentation problem, which can be very efficiently solved by proximal algorithms such as the Primal-Dual solver (Chambolle \& Pock 2011). In such a case, additional constraints can be naturally added to ensure smoothness and continuity of the identified boundaries between different geological units. The versatility of such solvers has recently led to the creation of a novel joint reconstruction and segmentation algorithm in the context of medical imaging Corona et al. (2019) that we seek to adapt to the problem of seismic inversion.

In this work, we propose a novel approach to computer-aided seismic interpretation, which aims to reconcile the classical principles of seismic interpretation to the underlying optimization problem that we define and solve. The interpreter input is here limited to the definition of a discrete number of expected geological units parametrised by their average acoustic impedance (AI) that we wish to ultimately extract from the seismic data. A joint inversion-segmentation of the input seismic data is then performed and both inverted acoustic properties and a segmented model of the subsurface are produced. As a by-product, the segmentation model is used as input to an automatic workflow for interpretation of seismic horizons at the boundaries between different zones. Examples on synthetic and field datasets show that our methodology can successfully produce high-resolution models of the subsurface from post-stack seismic data and geological priors and extract horizons at key geological boundaries defined by the interpreter in a fully automatic manner without any manual labelling or expensive upfront training,

\section{A PHILOSOPHICAL VIEW OF THE PROBLEM}

Traditionally, seismic data has been interpreted by humans looking at a variety of attributes (e.g., full stack, partial stacks, seismic attributes) and most commercial softwares are built around this principle. Whilst used in some cases to aid interpretation, elastic properties derived from seismic pre- or poststack inversion are generally not considered the main source of information against which to interpret horizons. Nevertheless, these properties are the physical reason behind what is observed within the seismic data: a large increase (or decrease) in acoustic impedance at a geological boundary produces 
a strong seismic response; conversely, a small change cannot trigger a response and so our ability to interpret such a geological boundary is in vain. We therefore ask ourselves whether a machine could be more successful at aiding interpretation if working directly with elastic properties other than the original seismic data? Or in other words, can we turn the problem of interpreting an horizon from following wiggles on seismic sections into a segmentation task for the acoustic (or elastic) properties in the geological formations of interest? In the following section, we introduce a mathematical formulation that translates this objection into implementable equations.

\section{MATHEMATICAL FORMULATION}

Inspired by the work of Corona et al. (2019) on joint reconstruction and segmentation in the context of medical imaging, we define a functional to invert seismic data for their acoustic impedance as well as to produce a segmentation of the subsurface into $N_{c}$ classes, each of which is associated with a specific range of impedance values. The underlying idea of inverting for these two parameters simultaneously is to inform the inversion with prior knowledge about the rock units of interest, whilst at the same time constraining this belief with the seismic measurements.

The functional for our specific problem can be written as

$$
(\mathbf{m}, \mathbf{V})=\underset{\mathbf{m}, \mathbf{V} \in C}{\operatorname{argmin}} J_{1}+\alpha J_{2}+\delta \widehat{J}_{3}+\beta J_{4}
$$

that we wish to minimize for $\mathbf{m}$ and $\mathbf{V}$ jointly. Here, $\mathbf{m}$ is a vector of size $N_{x} N_{z} \times 1$ that contains the natural logarithm of the acoustic impedance values in the area of the subsurface of interest, and $\mathbf{V}$ is a matrix of size $N_{c} \times N_{x} N_{z}$ whose columns contain the probability of each point in the subsurface to belong to each class. As part of the inversion, we further constrain each column of $\mathbf{V}$ to be in the unit Simplex, $C=\left\{\mathbf{V}_{i} \in \mathbb{R}^{+}: \sum_{j=1}^{N_{c}} V_{j i}=1\right\}, \forall i=1,2, \ldots, N_{z} N_{x}$. Note that by ensuring that the sum of the elements of each column of $\mathbf{V}$ is equal to 1 and every element is positive, we can interpret such values as the probability of each point in the subsurface belonging to a certain class. In this paper, we use the convention that $x_{i}$ is the $\mathrm{i}$-th element of a vector, $X_{i j}$ is the element from the $\mathrm{i}$-th row and $\mathrm{j}$-th column of a matrix, $\mathbf{X}_{i}$ represents the extraction of the $\mathrm{i}$-th column of a matrix, whilst $\mathbf{X}_{j}^{T}$ is the $\mathbf{j}$-th row of a matrix transposed into a column vector. Finally, $\alpha, \beta$, and $\delta$ are regularization parameters used to balance the importance of each individual term against the others, and are all selected relative to the unscaled first term $\left(J_{1}\right)$.

The four different contributions of the cost function $J$ are defined as follows:

- Datá misfit:

$$
J_{1}=\frac{1}{2}\|\mathbf{d}-\mathbf{G m}\|_{2}^{2}
$$


where $\mathbf{G}=\mathbf{W D}$ is the post-stack modelling operator composed of a first derivative operator $\mathbf{D}$ and a convolution operator $\mathbf{W}$ whose convolution kernel is the estimated wavelet $w$ divided by 2 , and $\mathbf{d}$ is post-stack seismic data of size size $N_{x} N_{z} \times 1$. This term ensures that the estimated model is consistent with the observed data.

- Blockiness of the acoustic impedence model:

$$
J_{2}=T V(\mathbf{m})
$$

where the Total-Variation norm is defined as $T V(\mathbf{x})=\|\nabla \mathbf{x}\|_{2,1}=\sum_{i=1}^{N_{x} N_{z}} \sqrt{\left(\mathbf{x}_{x}\right)_{i}^{2}+\left(\mathbf{x}_{z}\right)_{i}^{2}}$ where $\nabla: \mathbb{R}^{N_{x} N_{z}} \rightarrow \mathbb{R}^{2 \times N_{x} N_{z}}$ is the gradient operator that transforms a vector into a matrix whose rows contain the derivatives computed along the $\mathrm{x}-\left(\mathbf{x}_{x}\right)$ and $\mathrm{z}-\left(\mathbf{x}_{z}\right)$ directions, respectively. This terms contributes to creating a blocky model of the subsurface $\mathbf{m}$ with sharp discontinuities in correspondence of seismic events in the data d. Ultimately, this prior partially counteracts the lack of low and high frequencies in the seismic data and naturally extends the bandwidth of the inverted model.

- Segmentation:

$$
J_{3}=\sum_{j=1}^{N_{c}} \sum_{i=1}^{N_{x} N_{z}} V_{j i}\left(m_{i}-c_{j}\right)^{2}
$$

where $c_{j}$ is the $\mathbf{j}$-th element of the vector $\mathbf{c}$ of size $N_{c} \times 1$ that contains the acoustic impedance values associated to each class. This term has two different roles, depending whether the inversion process is focused on updating the property model $\mathbf{m}$ or the auxiliary segmentation model $\mathbf{V}$. Whilst keeping $\mathbf{V}$ fixed, this terms acts as a regularizer to the data misfit term driving the elements of the model vector $\mathbf{m}$ closer to the central value of the class they belong to. Whilst keeping $\mathbf{m}$ fixed, this term can be interpreted as a multi-label segmentation functional (see, e.g., Chambolle et al. (2012)) that updates each element $V_{i j}$ towards one when the corresponding $m_{i}$ is closer to the class centroid $c_{j}$ and towards zero otherwise.

- Blockiness of the segmentated model.

$$
J_{4}=\sum_{j=1}^{N_{c}} T V\left(\mathbf{V}_{j}^{T}\right)
$$

where the sum of the TV norms of each segmented model $\mathbf{V}_{j}^{T}$ associated to the class $c_{j}$ is minimized. Mathematically speaking this term enforces that the sum of the perimeters of each partition is minimal; from a physical point of view, it avoids the creation of multiple independent partitions for each class and favours the generation of larger partitions (Chambolle et al. 2012). As we will see in the next section, this term can be easily incorporated in the overall functional to minimise when working with proximal solvers, whilst it is not possible to do so when relying on greedy algorithms for segmentation such as the fuzzy c-means algorithm of Sun \& Li (2015). 


\subsection{Alternating minimization}

In order to solve the functional in equation 1 we note that, whilst non-convex in the joint argument $(\mathbf{m}, \mathbf{V})$, this cost function is convex in each individual variable space. In other words, whilst parameters $\mathbf{m}$ and $\mathbf{V}$ are nonlinearly related to each other (see equation 4 ) rendering the overall functional to be non-convex, if we keep one of the them fixed at the time, the functional becomes convex in the other parameter. This property allows solving the functional $J$ using a well-known splitting approach where the two resulting convex problems are minimised in an alternating fashion. Moreover, since solving TV-regularized problems can lead to a systematic loss of contrast in the estimated model (Benning \& Burger 2013), we replace both TV regularization terms with their generalized Bregman distance $\left(D_{T V}^{\mathbf{p}}\left(\mathbf{u}, \mathbf{u}^{\prime}\right)=T V(\mathbf{u})-T V\left(\mathbf{u}^{\prime}\right)-\left(\mathbf{u}-\mathbf{u}^{\prime}\right)^{T} \mathbf{p}-\right.$ Bregman (1967)) and Bregman iterations are introduced to solve each independent minimisation problem (Benning \& Burger 2013; Osher et al. 2005). This is a similar strategy to the one employed in the well-known Split-Bregman solver (Goldstein \& Osher 2009).

The overall algorithm reads as:

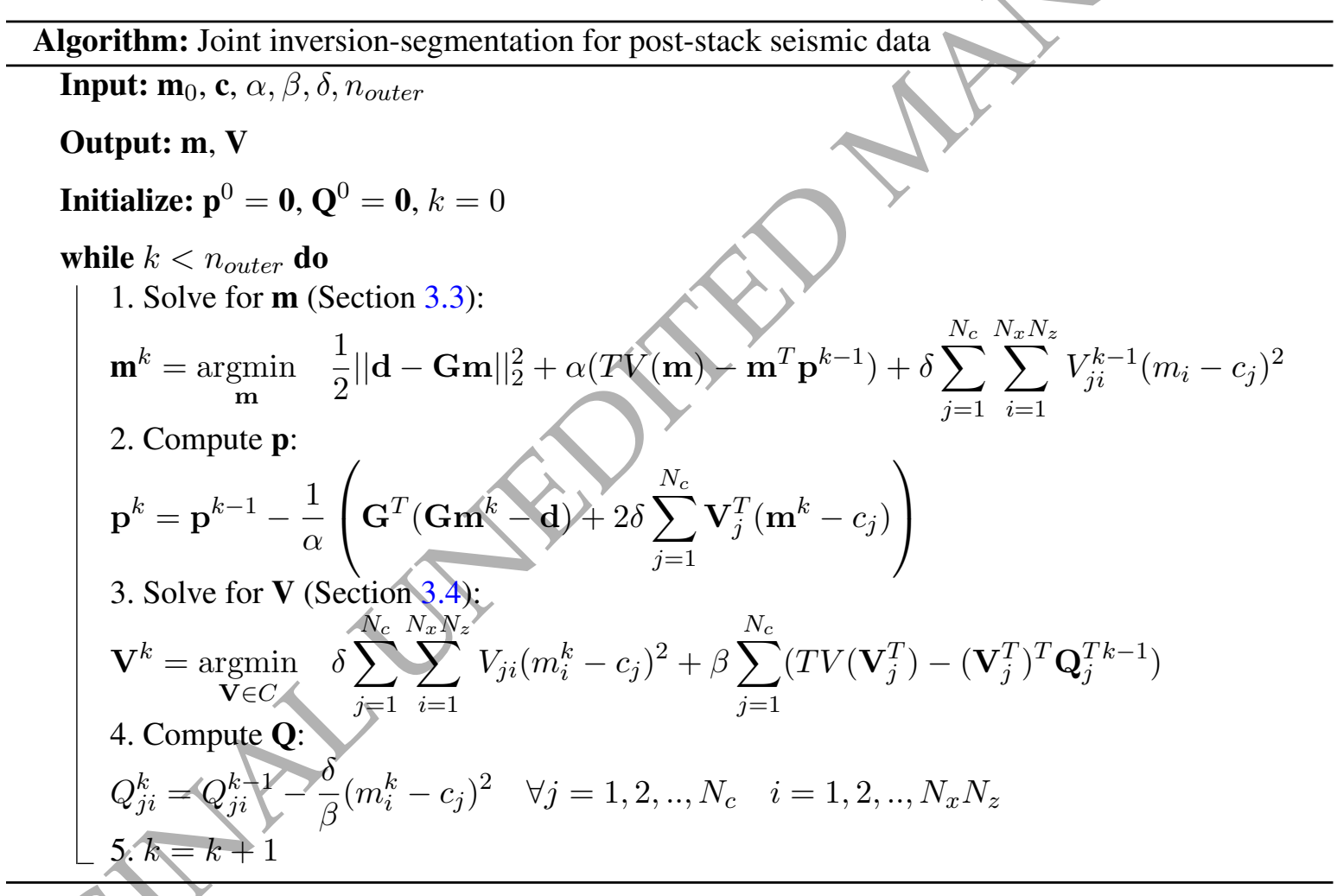

where $k$ indicates the current iteration, $n_{\text {outer }}$ is the chosen number of outer iterations (generally a few outer iterations suffice), $\mathbf{p}$ and $\mathbf{Q}$ are the sub-gradients of their corresponding Bregman distances. Note that whilst $\mathbf{p}^{k}$ and $\mathbf{Q}^{k}$ can be easily evaluated and that we write the equation for $\mathbf{Q}^{k}$ in an element- 
wise fashion for simplicity of notation, $\mathbf{m}^{k}$ and $\mathbf{V}^{k}$ require the solution of two optimization problems, which is here achieved using the Primal-Dual algorithm as detailed in the following section. Moreover, during the first outer iteration $(k=0)$, the segmentation term (equation 4 ) is omitted from step 1 . In later iterations $(k>0)$, this term acts as a soft constraints that drives the model parameters $\mathbf{m}$ towards their corresponding classes centroids $\mathbf{c}$.

The entire Algorithm is implemented using the open-source PyLops framework for large-scale optimization (Ravasi \& Vasconcelos 2020). More specifically, the PyLops ${ }^{1}$ library is used for the modelling operator of the post-stack seismic data $(\mathbf{G})$, whilst the proximal operators and solvers are provided by the PyProximal ${ }^{2}$ library.

\subsection{The Primal-Dual solver}

Whereas gradient-based methods are iterative optimization algorithms for solving unconstrained, smooth functionals, proximal algorithms can be viewed as their counterparts for non-smooth, possibly constrained versions of these problems. Broadly speaking, proximal algorithms are able to minimize any functional of the form $f(x)+g(x)$ where $g$ is a convex and differentiable function, whilst $f$ is convex but possibly non-differentiable function; we will soon see this to be the case for the functionals in steps 1 and 3 our Algorithm.

Minimisation is performed in an iterative fashion where each iteration generally relies on the ability of evaluating the gradient of the smooth function $g$ and the so-called scaled proximal operator of the scaled non-smooth function $\tau f$ (with $\tau>0$ ) that is defined as (Parikh 2013):

$$
\operatorname{prox}_{\tau f}(\mathbf{u})=\underset{\mathbf{x}}{\operatorname{argmin}} f(\mathbf{x})+\frac{1}{2 \tau}\|\mathbf{x}-\mathbf{u}\|_{2}^{2}
$$

Evaluating the proximal operator involves solying an optimization problem, which intuitively compromises between minimizing $f$ and finding a solution close to $\mathbf{u}$. When used in the context of non-convex optimization, the proximal operator of a non-differentiable functional $f$ can be seen as the equivalent to a gradient step of a differentiable function in convex optimization where the sub-gradient of $f$ is used instead of the gradient (as the former is not uniquely defined for a non-differentiable function). Luckily, as shown in the Appendix 1, closed form solutions exist for many commonly used proximal operators, meaning that these sub-problems can be solved very quickly with fast specialized methods.

The Primal-Dual algorithm of Chambolle \& Pock (2011) is a special type of proximal solver that shows $O(1 / N)$ rate of convergence in finite dimensions. It works by recasting any problem of this

\section{2}

http://github.com/PyLops/pylops

2 http://github.com/PyLops/pyproximal 
kind:

$$
\underset{\mathbf{x}}{\operatorname{argmin}} f(\mathbf{K} \mathbf{x})+\sum_{i} \mathbf{x}^{T} \mathbf{z}_{i}+g(\mathbf{x})
$$

into its primal-dual equivalent (i.e., a saddle-point problem):

$$
\underset{\mathbf{x}}{\operatorname{argmin}} \underset{\mathbf{y}}{\operatorname{argmax}} \mathbf{y}^{T}(\mathbf{K} \mathbf{x})+\sum_{i} \mathbf{z}_{i}^{T} \mathbf{x}+g(\mathbf{x})-f^{*}(\mathbf{y})
$$

where $f$ and $g$ are convex (possibly non-smooth) functionals, $f^{*}$ is the convex conjugate of $f$ and $\mathbf{K}$ is a linear operator that maps the vector $\mathbf{x} \in R^{m}$ into a vector $\mathbf{y} \in R^{n}$.

A series of iterations is then introduced in order to obtain convergence at the saddle point:

$$
\left\{\begin{array}{l}
\mathbf{y}^{k+1}=\operatorname{prox}_{\mu g^{*}}\left(\mathbf{y}^{k}+\mu \mathbf{K} \overline{\mathbf{x}}^{k}\right) \\
\mathbf{x}^{k+1}=\operatorname{prox}_{\tau f}\left(\mathbf{x}^{k}-\tau\left(\mathbf{K}^{H} \mathbf{y}^{k+1}+\sum_{i} \mathbf{z}_{i}\right)\right) \\
\overline{\mathbf{x}}^{k+1}=\mathbf{x}^{k+1}+\theta\left(\mathbf{x}^{k+1}-\mathbf{x}^{k}\right)
\end{array}\right.
$$

where $\theta \in[0,1](\theta=1$ will be used in our work), $\tau$ and $\mu$ represent the step-lengths of the two subgradients. To ensure convergence $\tau \mu L^{2}<1\left(L^{2}=\|\mathbf{K}\|_{2}^{2}=\lambda_{\max }\left(\mathbf{K}^{H} \mathbf{K}\right)\right.$ - i.e., the spectral radius of the linear operator.

\subsection{Inversion}

We start by analysing step 1 in the Algorithm and showing that it is possible to write it in the form of equation 7. Specifically, we write $\mathbf{K}=\nabla, \mathbf{z}=-\alpha \mathbf{p}^{k-1}, f=\|\|_{2,1}$, and $g=\frac{1}{2}\|\mathbf{d}-\mathbf{G m}\|_{2}^{2}+$ $\delta \sum_{j=1}^{N_{c}} \sum_{i=1}^{N_{x} N_{z}} V_{j i}^{k-1}\left(m_{i}-c_{j}\right)^{2}$. For simplicity, we rearrange some of the terms in $g$ such that we can write it as $g=\frac{1}{2}\|\hat{\mathbf{d}}-\hat{\mathbf{G}} \mathbf{m}\|_{2}^{2}$ :

$$
\hat{\mathbf{V}}=\left[\begin{array}{c}
\operatorname{diag}\left\{\sqrt{\mathbf{V}_{1}^{T}}\right\} \\
\cdots \\
\operatorname{diag}\left\{\sqrt{\mathbf{V}_{N_{c}}^{T}}\right.
\end{array}\right], \hat{\mathbf{c}}=\left[\begin{array}{c}
\sqrt{\mathbf{V}_{1}^{T}} c_{1} \\
\cdots \\
\sqrt{\mathbf{V}_{N_{c}}^{T}} c_{N_{c}}
\end{array}\right], \hat{\mathbf{G}}=\left[\begin{array}{c}
\mathbf{G} \\
\sqrt{2 \delta} \hat{\mathbf{V}}
\end{array}\right], \hat{\mathbf{d}}=\left[\begin{array}{c}
\mathbf{d} \\
\sqrt{2 \delta} \hat{\mathbf{c}}
\end{array}\right]
$$

where the square root acts on each individual element of the vector it is applied to. The evaluation of the proximal operator of $f$ and $g$ is discussed in Appendix A.

\subsection{Segmentation}

Segmentation is obtained as a result of step 3 in the Algorithm. In order to transform such a functional into one that is suitable for the Primal-Dual solver, we rewrite it as follows:

$$
\mathbf{v}^{k}=\underset{\mathbf{v} \in C}{\operatorname{argmin}} \mathbf{v}^{T}\left(\delta \mathbf{g}-\beta \mathbf{q}^{k-1}\right)+\beta \sum_{j=1}^{N_{c}} T V\left(\mathbf{V}_{j}^{T}\right)
$$


where $\mathbf{v}=V e c(\mathbf{V})$ and $\mathbf{q}=V e c(\mathbf{Q})$ are the vectorized version of $\mathbf{V}$ and $\mathbf{Q}$, respectively, obtained by concatenating their rows into a vector. Moreover, we define the weighting vector $\mathrm{g}=$ $\left(\left(\mathbf{m}^{T}-c_{1}\right)^{2}, . .,\left(\mathbf{m}^{T}-c_{N_{c}}\right)^{2}\right)^{T}$, where the superscript 2 is used to indicate the squared difference between each element of the vector $\mathbf{m}$ and the chosen coefficient $c_{j}$. In its current form, equation 11 can be shown to be on the form of equation 4 . This is achieved by writing $\mathbf{K}=\nabla, \mathbf{z}=\delta \mathbf{g}-\beta \mathbf{q}^{k-1}$, $f=\|\|_{2,1}$, and $g=i_{C}$ i.e., the indicator of the Simplex function.

\section{HORIZON EXTRACTION}

Horizon extraction is performed independently for each of the segmentation classes, as illustrated in Figure 1. First, each class probability is binarised based on the highest probability at each spatial location, identifying the areas which are allocated to that class after the segmentation is complete. The resulting binary class images undergoes a cleaning step. Morphological opening, a popular image processing technique for removing small objects from an image while preserving the shape and size of larger objects, is applied here. This technique ultimately converts spurious foreground pixels in a fixed sized window (here $3 \times 3$ ) to the dominant background pixel value. The terminology of foreground and background here represents the areas in the binary class image that have either been detected as that class or not.

Subsequently, the previously defined Total Variation norm is computed on the cleaned class image. Such a measure is frequently used by edge detection algorithms as it isotropically enhances the edges of objects. This computation identifies the boundaries of areas belonging to the class, i.e., the edges of the foreground objects, such that pixels with a high TV value are indicative of a boundary. Finally, a threshold is applied where every pixel above the threshold is determined to be a potential horizon point. Throughout the numerical experiments we observe that a value between 0.2-0.4 is generally effective, and that slightly lower values (e.g., 0.2) should be preferred as the noise in the data increases.

Once detected, neighbouring horizon points are joined in a left-right, top-bottom fashion. Beginning from the top, most-left horizon point, $\left(x_{0}, z_{0}\right)$, neighbouring horizon points are identified within the limits $x_{0}<x<x_{0}+n_{\text {search }}$ and $z_{0}-n_{\text {search }}<z<z_{0}+n_{\text {search }}$, where $n_{\text {search }}$ indicates the search area. If there are multiple neighbouring points satisfying the TV threshold, the one with the highest TV value is selected as the next horizon point and the same neighbour search begins again from the new points, $\left(x_{1}, z_{1}\right)$. The neighbour search continues until no points are detected in the neighbour space, concluding that line. The $n_{\text {search }}$ parameter is usually selected to be between 2-4, where higher values should be preferred for noisy data where we may expect rugged edges in the TV-thresholded classes.

At this point, a new neighbour search begins from the highest, most-left remaining horizon points 
to create another line. The colours and separated lines in the connect image of Figure 1 illustrate the results of the neighbour search step for this conceptual example. As soon as the neighbour search is exhausted, the lines are labelled using the segmented image to determine the class above and class below the lines, as illustrated in the label image of Figure 1. Three options exist for labelling the lines depending on the complexity of the geology and the homogeneity of the medium above and below the line. The labelling options are: only consider the class above, only consider the class below, or consider both classes above and below.

Similar to the connect step performed on a pixel level, a secondary combining procedure is performed on the labelled lines considering any lines that begin within a specified window from the ending of the previous line. Due to the smaller number of lines available to be joined, in comparison to the pixels in the connect step, a larger window can be considered allowing joining of points previously too far apart, as illustrated by the combining of the $3 A B$ lines in Figure 1. For lines that are too far apart, such as $B C$, these will remain as separate lines to avoid any artefacts being generated in the regridding step.

Finally, a regridding step is performed for each identified horizon line. For continuous horizons, such as $A B$, the regridding procedure ensures a single depth value per horizontal location. On the other hand, when horizons present contiguous gaps larger than a specified threshold (e.g., $B C$ ), the regridded horizon is further masked (i.e., NaN values are placed in place of the gaps) to avoid the creation of spurious connections. As a result, all of the identified horizons span the full lateral extent of the model. Note that, once the horizons have been labelled and extracted for each class, duplicate horizons may exist when a single horizon has been identified as the top horizon from one class image and as the bottom horizon from another class image. However, as TV image values vary from one class image to another, the duplicate horizons are unlikely to be identical. For example in the case of the $B C$ horizon, the fault may have been detected when the class image represented the medium below the $B C$ horizon. Duplicate horizons arising from the above mentioned scenario are generally undesirable, although there may be special scenarios where horizons have been given the same label based on their neighbouring classes where it is desirable to retain both, such as highly-layered settings.

A final horizon selection procedure is therefore performed for each group of horizons with the same label as follows:

(i) All horizons are initially set as "potential-horizons".

(ii) The longest horizon, i.e. with the fewest $\mathrm{NaN}$ values, is identified, removed from the list of potential horizon lines, and added to the list of selected horizons.

(iii) For each remaining horizon line, any shared points on the $\mathrm{x}$-axis are compared and the average depth difference between the points is computed. 

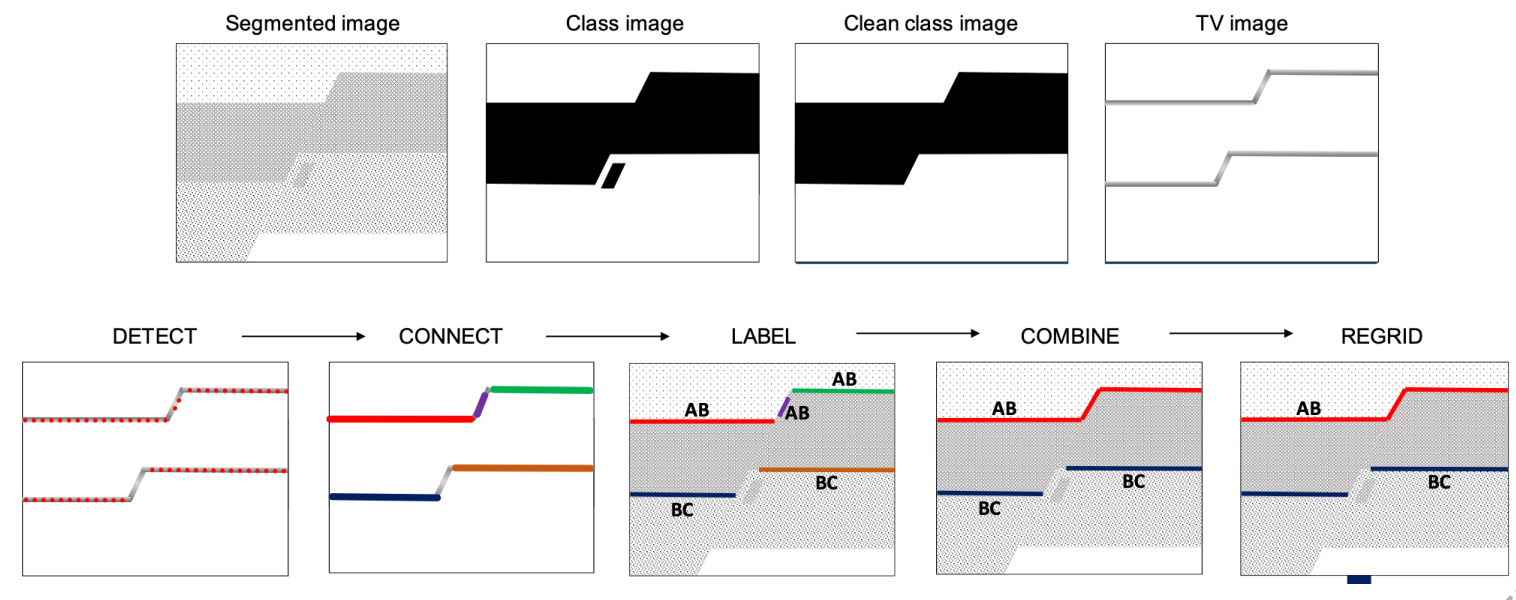

Figure 1. Workflow for the extraction of horizon lines from a single class. The segmented image is the starting point of the horizon extraction procedure from which a binary class image is computed prior to cleaning and performing a TV calculation to determine the edges of the class object. Neighbouring edge points are connected prior to being labelled based on surrounding classes. Finally similarly labelled lines are combined before being regridded to the full horizontal, spatial sampling of the model. The blue line on the $\mathrm{X}$-axis of the regridding step illustrates where horizontal values are $\mathrm{NaN}$.

(iv) If the difference is less than a given threshold the horizon is considered a duplicate of the longest horizon and is removed from the list of potential horizon lines.

(v) If the difference is larger than the threshold, the horizon line remains on the potential horizon list and is still considered a "potential-horizon".

(vi) Steps 2-5 are repeated until there are no horizons left on the potential horizon list, i.e., until all horizons lines are either selected or rejected.

The selected horizons, alongside the inverted and segmented models, ultimately represent the final outputs of our algorithm.

\section{EXAMPLES}

In this section, we apply our methodology to two synthetic examples and a 2D line of the open-source Volve dataset.

\subsection{Simple model}

The first example considers a simple subsurface model composed of a number of horizontally stacked layers offset by a normal fault and serves the purpose of discussing the introduction of a segmentation step in a standard seismic inversion workflow and its usage in the context of automatic horizon tracking. Because of its simplicity, we show here that a single step of the proposed inversion scheme 
(a)

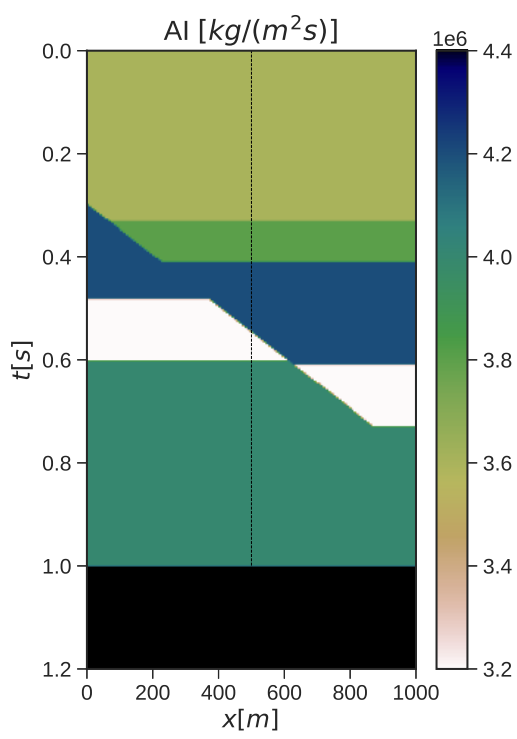

(b)

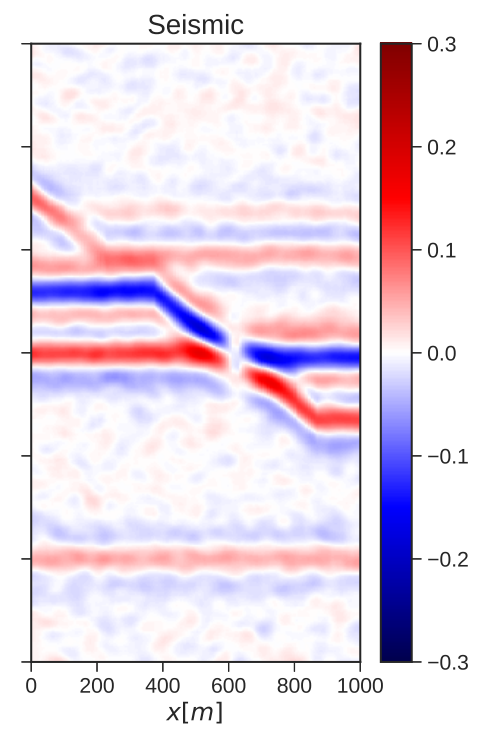

(c)

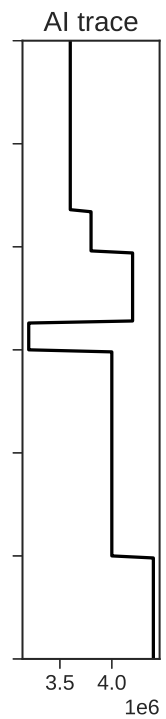

Figure 2. a) Acoustic impedance model, b) noisy seismic data, and c) AI profile at well location (shown by the dashed black line in panel a).

suffices to obtain a satisfactory segmentation of the model into different zones and for the tracking algorithm to extract all the horizons of interest. Nevertheless, despite its apparent simplicity, this model presents offset horizons which are historically challenging to track in seismic data.

Figure 2a displays the acoustic impedance model composed of six formations with different properties. For the sake of this exercise, we assume that a well is drilled in the middle of the model, such that all formations are sampled. The corresponding acoustic impedance profile is displayed in Figure 2c. The class vector $\mathbf{c}$ is therefore chosen to be a $6 \times 1$ vector whose elements are the different AI values extracted from the vertical profile. The post-stack seismic data (Figure $2 b$ ) are modelled using the $\mathbf{G}$ operator discussed above and a $8 \mathrm{~Hz}$ Ricker wavelet. Note that throughout the paper we define the vertical axis in terms of two-way traveltime for consistency with the field data example. Finally colored noise is added to the synthetic dataset with signal-to-noise ratio equal to $15 \mathrm{~dB}$ : the noise model is created by smoothing a white noise realization along both the spatial and time axes.

To begin with, the noisy dataset $\left(\mathbf{d}_{\mathbf{n}}=\mathbf{G m}+\mathbf{n}\right)$ is inverted for the underlying acoustic impedance model (m) using a number of approaches:

- Least-squares regularized inversion: $J_{L 2}=\left\|\mathbf{d}_{\mathbf{n}}-\mathbf{G m}\right\|_{2}^{2}+\epsilon\|\mathbf{m}\|_{2}^{2}+\alpha\left\|\nabla^{2} \mathbf{m}\right\| \|_{2}^{2}$ where $\nabla^{2}$ is the Laplacian operator. The second term in the functional is the standard Tikhonov regularization, whilst the third term enforces smoothness in the solution. The model $\mathbf{m}$ can be estimated by using any iterative solver for smooth, convex functionals; here we use LSQR (Paige \& Saunders 1982) with 
(a)

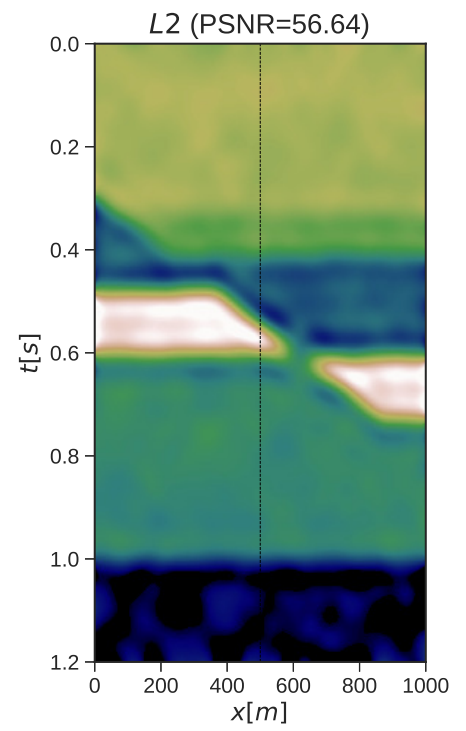

(b)

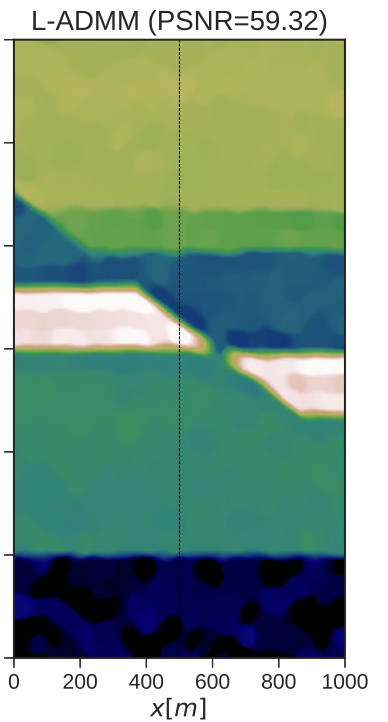

(c)

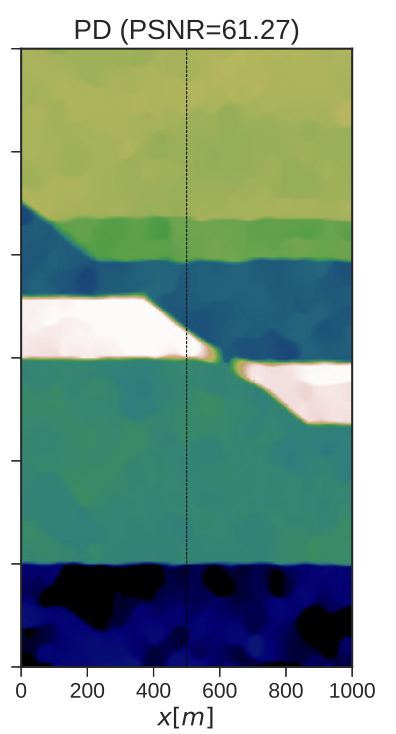

(d)

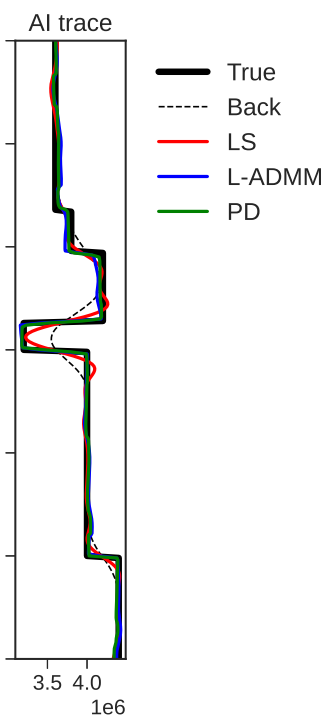

?

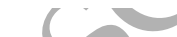

Figure 3. Inverted models via a) Least-squares regularized inversion, b) Isotropic TV-regularized inversion with L-ADMM solver, and c) Isotropic TV-regularized inversion with Primal-Dual solver.

a smooth version of the model as starting guess. This results serves as a baseline as it represents the approach routinely used for post-stack inversion of seismic data.

- Linearized Alternating Direction Method of Multipliers (L-ADMM) with isotropic TV regularization (Boyd et al. 2011): $J_{L-A D M M}=\frac{1}{2}\left\|\mathbf{d}_{\mathbf{n}}-\mathbf{G m}\right\|_{2}^{2}+\alpha T V(\mathbf{m})$.

- Primal-dual algorithm with isotropic TV regularization (Chambolle \& Pock 2011): $J_{P D}=J_{L-A D M M}$

In Figure 3 the different inversion results are compared and for each solution we compute the Peak Signal-to-Noise Ratio $\left(P S N R=10 \log _{10}\left(N_{x} N_{z} \max (\hat{\mathbf{m}})^{2} /\|\mathbf{m}-\hat{\mathbf{m}}\|_{2}^{2}\right)\right)$, where $\mathbf{m}$ is the true model and $\hat{\mathbf{m}}$ is the estimated model). First, we observe that whilst computationally cheaper than the other methods, least-squares inversion suffers from a major limitation: the retrieved model shows smooth transitions between different zones and therefore the sharp boundaries are not recovered. Whilst this is well-known limitation of such a method, it is particularly detrimental for our subsequent tasks of segmentation and horizon tracking as shown below. On the other hand, both the L-ADMM and Primal-Dual algorithms are able to recover a model with sharp boundaries and achieve an overall higher PSNR. Finally, we note that the Primal-Dual solver produces an estimate with slightly higher PSNR than the L-ADMM algorithm whilst being comparable in terms of computational cost.

The different inverted models are now used as input to the segmentation step. In mathematical terms, this is equivalent to solving a simplified version of step 2 in the Algorithm which contains the TV norm instead of the Bregman TV norm - in other words, where $\mathbf{Q}$ is set to zero. Figures 4 and 5 show the results of the segmentation for the model produced by least-squares and Primal-Dual 
inversions, respectively. In both cases, panel (a) shows the true segmented model, where each image pixel is assigned to the label of its class. In panel $b$ the binarised version of the estimated matrix $\mathbf{V}$ is displayed; here binarised means that the index of the highest value of each column of $\mathbf{V}$ is selected. The segmentation result from the least-squares model presents an evident problem inherited from the smooth transitions across interfaces observed in the inverted model; green rings appear all around the red class due to the fact that the model parameter of the green class lies in between that of the red and yellow classes. This becomes even more evident when observing the probabilities of each class in a vertical pillar in the middle of the model (Figure 4d). In this plot, the probabilities of each class at each depth level are stacked along the x-axis. For example, we observe that only one class (i.e., light green) is predicted in the shallower section with probability of $100 \%$. This probability diminishes towards $10 \%$ for times closer to the first horizon as the probability of another class (i.e., blue) starts to increase to about $90 \%$ (at a time of $0.35 \mathrm{~s}$ ). The absence of one or more colors at certain depth levels is indicative of zero probability of occurrence of the associated class. Finally, when attempting to track horizons, the presence of such rings affects the overall quality of the estimated horizons. Moreover, some of the erroneously tracked horizons do not conform with the layering knowledge provided by the input well log.

On the other hand, the segmentation results obtained when using the model estimated via the Primal-Dual solver are much more accurate. Note also that the estimated probabilities present sharper transitions between the different zones. This greatly benefits the subsequent step of horizon tracking which returns the five main horizons with a very high degree of precision. Overall, we can conclude that the process of identifying seismic horizons by means of tracking of the edges of closed shaped polygons seems to be an appealing alternative to the more commonly used approach of working directly in the seismic data domain.

\subsection{Hess model}

Our second example is based on a modified version of the P-wave velocity of the SEG Hess VTI model (Figure 6a). This model is chosen for two reasons: first, we want to investigate the ability of our method to deal with stratigraphies that are interrupted by intrusions such as the salt body present in this model. Second, we are interested to assess the importance of our joint inversion-segmentation approach in the presence of very strong impedance contrasts such as those caused by the salt body.

To begin with, we consider once again the case where we have complete knowledge of the different zones in the model and their corresponding acoustic impedance values. Similar to the previous example, the post-stack seismic data (Figure 6b) are modelled using the $\mathbf{G}$ operator with a $8 \mathrm{~Hz}$ Ricker wavelet. Colored noise with signal-to-noise ratio equal to $11.6 \mathrm{~dB}$ is finally added. 
(a)

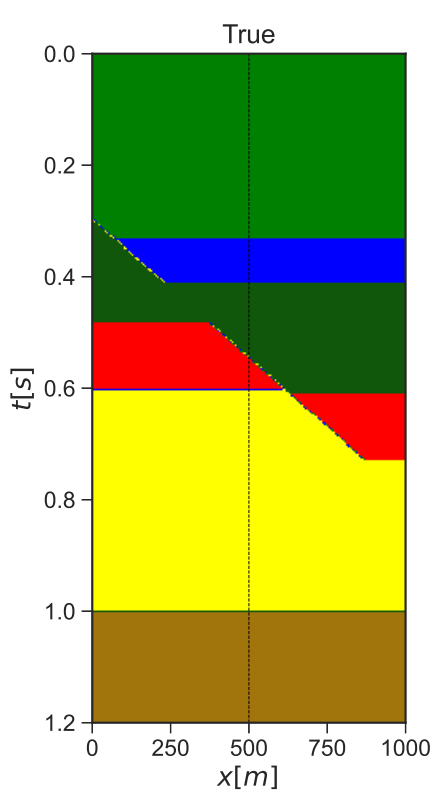

(b)

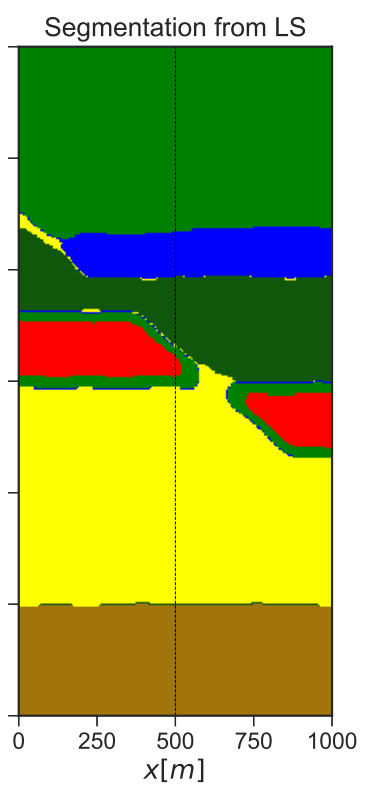

(c)

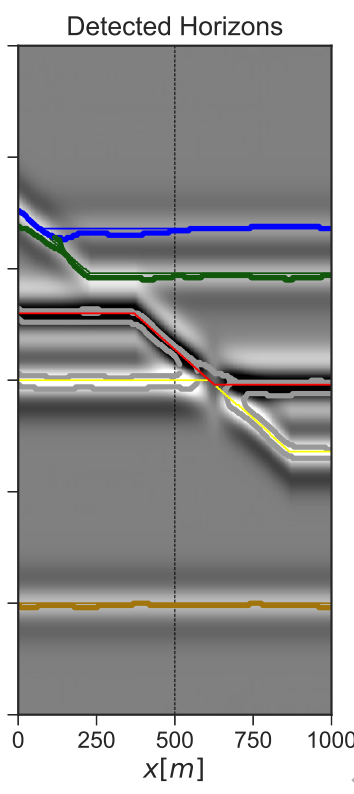

(d)

Probs.

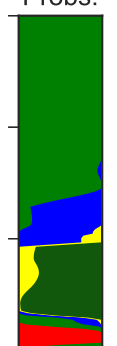

Figure 4. a) True and b) estimated segmentation from least-squares inverted model, respectively. c) Tracked horizons overlaid to the noise free seismic dataset (thin lines: true horizons, thick lines: tracked horizons). d) Probabilities for each class at well location (i.e., $N_{z}$ columns of $\mathbf{V}$ at the horizontal index in the middle of the model.

(a)

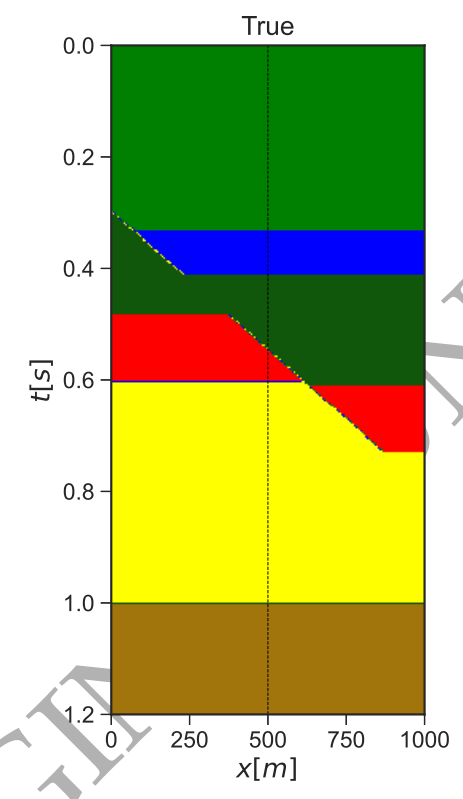

(b)

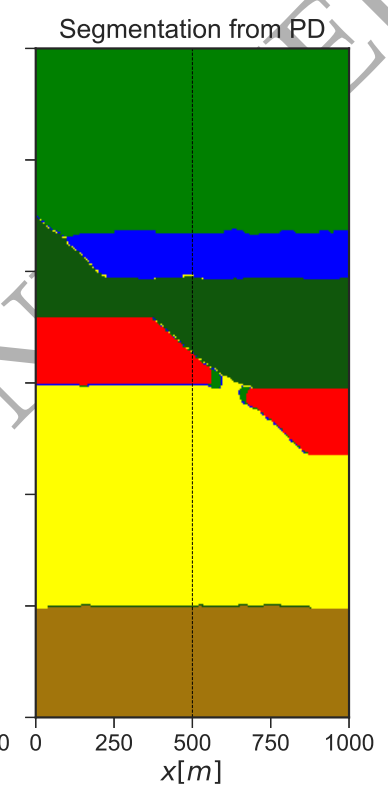

(c)

Detected Horizons

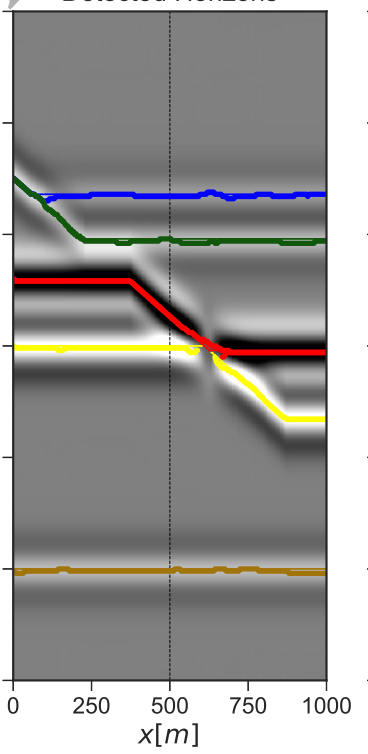

(d)

Probs.

Figure 5. Same as Figure 3 with the Primal-Dual model used as input for segmentation. 
(a)

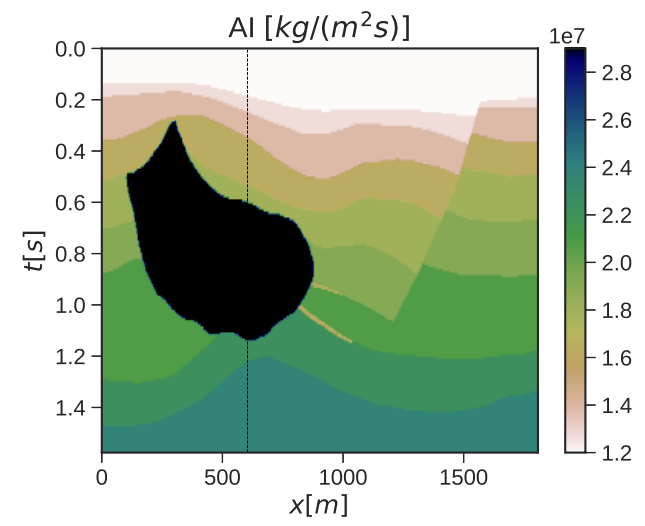

(b)

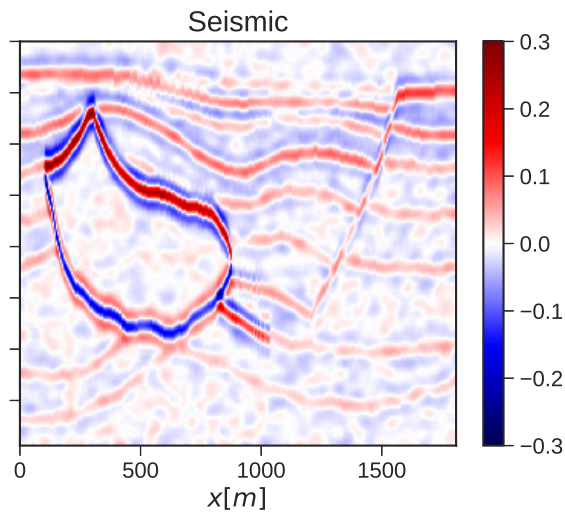

(c)

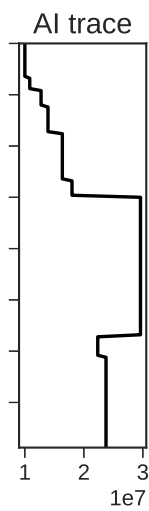

Figure 6. a) Acoustic impedance model, b) noisy seismic data, and c) AI profile at reference location indicated by dashed black line in panel a).

Figure 7 shows the estimated model for three different solvers: LSQR (used for the least-squares regularized functional), L-ADMM and PD (used for the TV regularized function). Once again, the inversion of noisy data benefits from use of the isotropic TV norm which regularizes the solution and produces sharp discontinuities at the edges of the model. Nevertheless, a common feature of the three inverted models is represented by the inaccurate estimate of the acoustic impedance of the salt body, which is underestimated by all of the tested algorithms. This is not surprising as the background model is fairly far from the true solution, especially towards the edges of the salt body, and the frequency content of the seismic data does not allow to recover such a gap even when a strong regularization such as the isotropic TV norm is included in the functional to optimize. A similar conclusion can be drawn by looking at the inverted AI profiles at the reference location (Figure 8a).

Despite the observed inaccuracies in the retrieved acoustic impedance model, segmentation and horizon tracking still produce satisfactory results when applied to the Primal-Dual model in Figure 7c. Note that, when compared to the true model (Figure 9a), most of the zones have been segmented precisely (Figure 9b); only the shallowest and two deepest zones are quite noisy, possibly due to the weak impedance contrasts affected by noise in the data (Figure 6b). A similar conclusion can be drawn for the outcome of the horizon tracking step (Figure 9c) where most of the horizons overlay almost perfectly with their true counterpart apart from those related to the poorly recovered zones.

The joint inversion-segmentation algorithm proposed in the previous section is now employed to evaluate whether constraining repeated steps of inversion with previously segmented zones could further improve the results in Figures 7 and 9. The retrieved model in Figure 10a after 4 outer iterations of our Algorithm does indeed more closely resemble the true model. This is especially the case for the salt body, whose absolute value is not underestimated in this case (Figure 8a), as well as the overall 
(a)

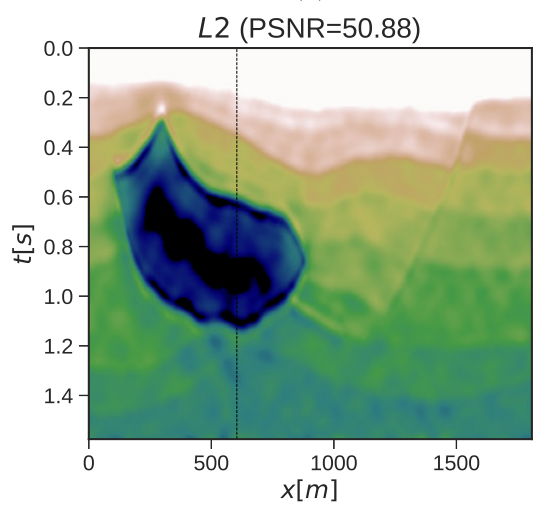

(b)

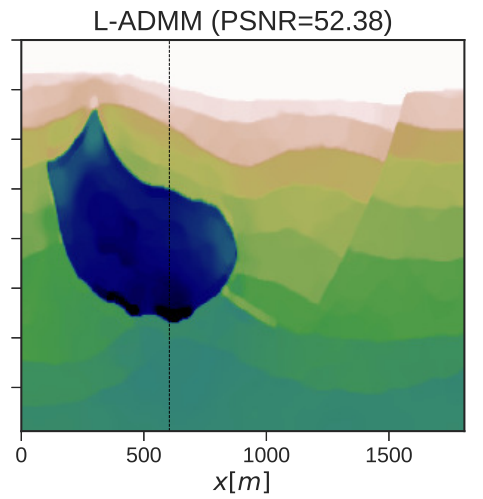

(c)

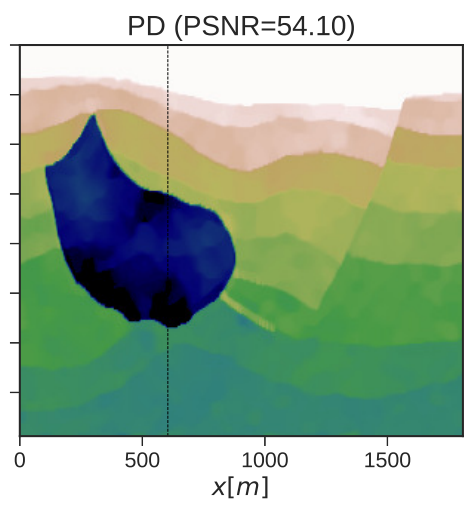

Figure 7. Inverted models via a) Least-squares regularized inversion, b) Anisotropic TV-regularized inversion Split-Bregman solver, and c) Isotropic TV-regularized inversion Primal-Dual solver.

continuity of the different layers. This is the consequence of the fact that the first step of segmentation is used as soft constraint to the second step of inversion (step 1 in the Algorithm), whose output is in turn used to drive the second step of segmentation (step 3 in the Algorithm), and so on and so forth. The outcome of the last segmentation step (Figure 10b) is also clearly more accurate than the one produced by directly using the Primal-Dual model: we observe a better continuity of the top interface of the shallowest zone as well as closer resemblance to the true classes in the deeper parts of the model.

(a)

(b)

(c)
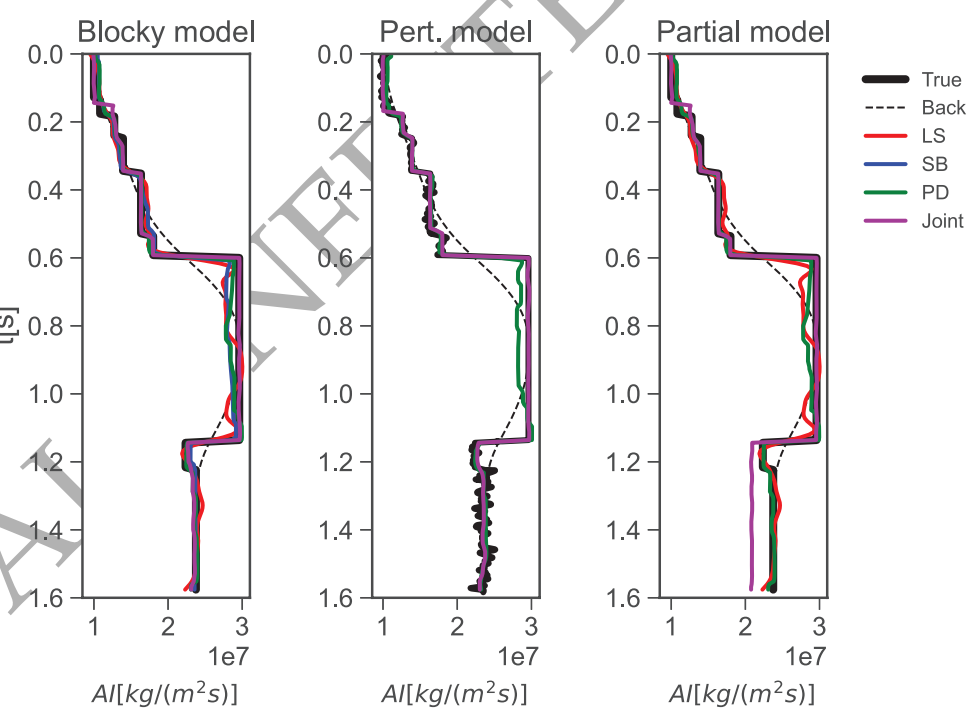

Figure 8. Acoustic impedance estimates for the different inversion algorithms at reference location for Hess dataset. a) Blocky model in Figure 6a, b) Model with small scale perturbations in Figure 11a, and c) Model with short well in Figure 12a. 
(a)

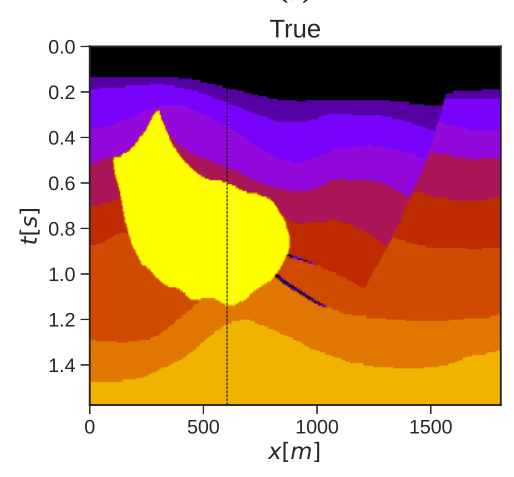

(b)

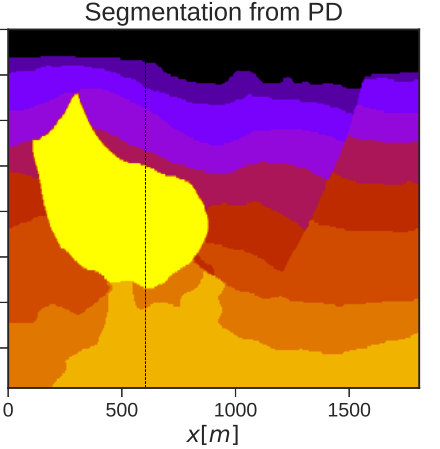

(c)

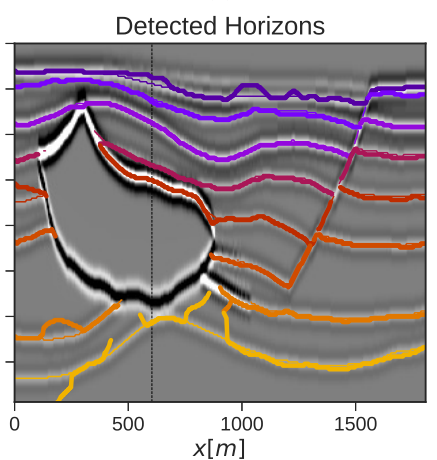

(d)

Probs.

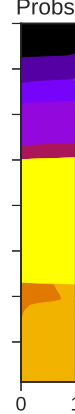

Figure 9. a) True and b) estimated segmentation from Primal-Dual model, respectively. c) Tracked horizons overlaid to the noise free seismic dataset (thin lines: true horizons, thick lines: tracked horizons). d) Probabilities for each class at reference location (i.e., a single column of $\mathbf{V}$ at the index in the middle of the model.

Similarly, the tracked horizons (Figure 10c) are of overall higher quality especially on the left of the salt body and in the deeper part of the model.

A second scenario is now evaluated. The acoustic impedance model in Figure $6 \mathrm{a}$ is modified by adding small scale fluctuations to the macro model composed of a limited number of acoustic impedance values. Such small scale variations are clearly visible in the log data (Figure $8 b$ ) and further complicate the overall seismic response (Figure 11d). In this case, we assume availability of two wells that intersect all of the geological units; the definition of our classes $\mathbf{c}$ is obtained by means of clustering of the acoustic impedance values from such wells. Inevitably, the estimated values of $\mathbf{c}$ slightly different from those in the first example. We clearly do not expect our inversion to be able to

(a)

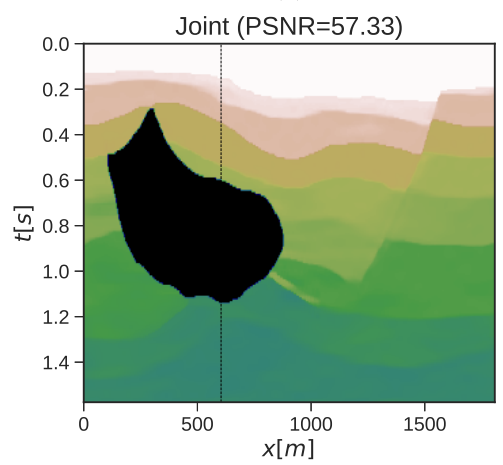

(b)

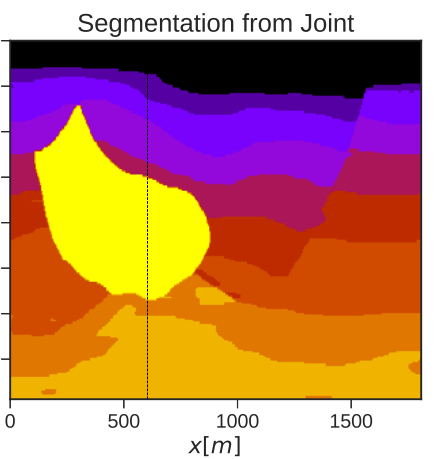

(c)

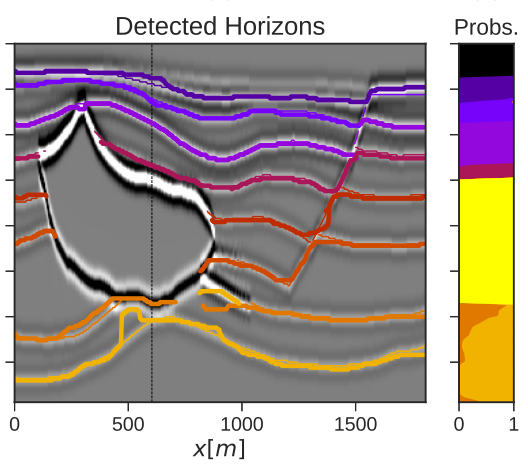

(d) Probs.

Figure 10. a) Model and b) segmentation from joint inversion scheme. c) Tracked horizons overlaid to the noise free seismic dataset (thin lines: true horizons, thick lines: tracked horizons. d) Probabilities for each class at reference location (i.e., a single column of $\mathbf{V}$ at the index in the middle of the model. 
recover the small fluctuations given that they are outside of the bandwidth of the signal: on the other hand, this examples serves the purpose of testing the sensitivity of our algorithm to more complex and realistic models of the subsurface as well as slightly incorrect c values. Panels c, e, and $\mathrm{f}$ in Figure 11 show that the different steps of our joint inversion are robust to small scale variations in the model and the results are overall very similar to those of the ideal case with a blocky macro model. Whilst all the horizons are successfully tracked in the segmented model, some spurious events are also reconstructed as part of the deeper horizon. This is the direct consequence of small blobs in the deeper layer of the segmented model (Figure 11e) which lead our tracking algorithm to identify those lines as part of the same horizon group as the deepest horizon because they border the same pair of classes as the larger horizon does.

Finally, we investigate the robustness of our algorithm with respect to partial knowledge of the different classes. As shown in Figure 12, a vertical well is now assumed to penetrate only some of the layers in the model: as a consequence, the classes vector $\mathbf{c}$ only contains seven elements to which we add an eighth based on the assumption that we are aware of the presence of the salt body and have a good estimate of its acoustic impedance. Whilst our algorithm fails to track the two deepest interfaces, both the inversion and horizon tracking of the shallow subsurface is still satisfactory and not affected by the lack of knowledge of the deeper part of the model. On the other hand, as shown in Figures 12a and Figures 8c, the acoustic impedance estimates in the deeper section are underestimated as a consequence of the fact that our joint algorithm tends to drive acoustic impedance values at each location closer to those of the class that has been selected in the previous iteration.

\subsection{Volve dataset}

Volve is an oil field located in the central part of the North Sea, five kilometres north of the Sleipner Øst field. Volve produced oil from sandstone of Middle Jurassic age in the Hugin Formation, with the main reservoir located at a depth of approximately 2,700-3,100 metres. The field was shut down in 2016, with the facility removed in 2018, and all historical subsurface and production data was made available by Equinor ín June 2018.

In this section, our joint inversion-segmentation algorithm is applied to a $2 \mathrm{~d}$ section of the PSDM full stack dataset from the ST10010ZC11 survey (Figure 13a). Since interpretation has been carried out in time, the time version of this dataset is used in our example. The section is extracted along the NO/15-919BT2 well and further extended to the East of the well as shown on top of a time map of the BCU time surface (Figure 13b). Figure 13c shows the acoustic impedance log converted into two-way traveltime (TWT) using the available checkshot profile. The 3 key interpreted horizons are overlain on the seismic section in Figure 13a and their corresponding well markers are shown in Figure 13c. 

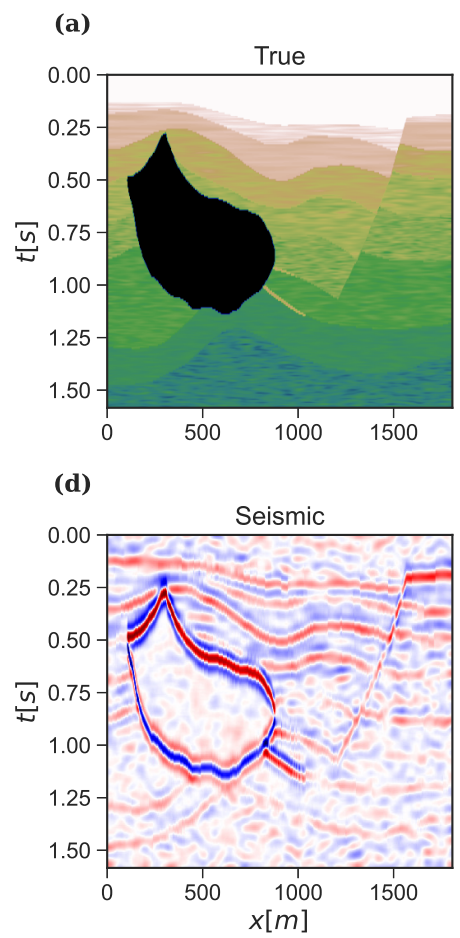

(b)

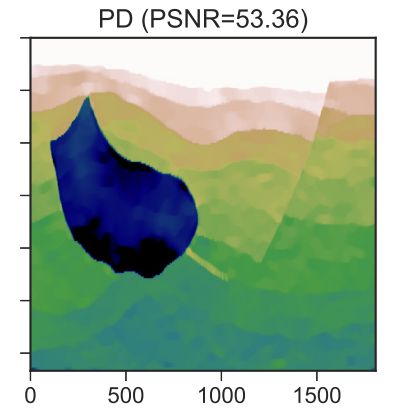

(e)

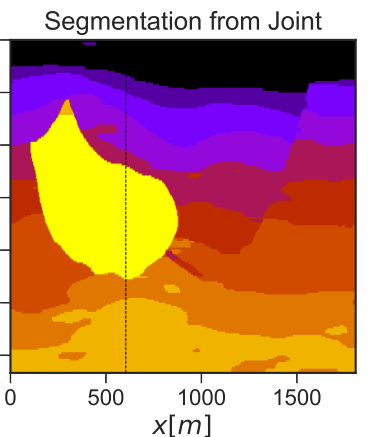

(c)

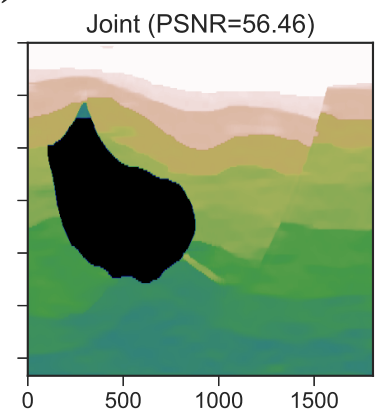

(f)

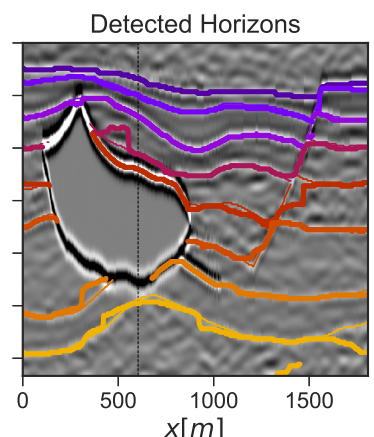

Figure 11. Results for model with small scale variations. a) True model, b) model from Primal-dual inversion, c) model from joint inversion scheme, d) noisy data, e) segmentation from the joint inversion scheme, f) tracked horizons overlain on the noise-free seismic dataset (note that noisy features are due to small scale AI perturbations in the model), and g) probabilities for each class at reference location.

(a)

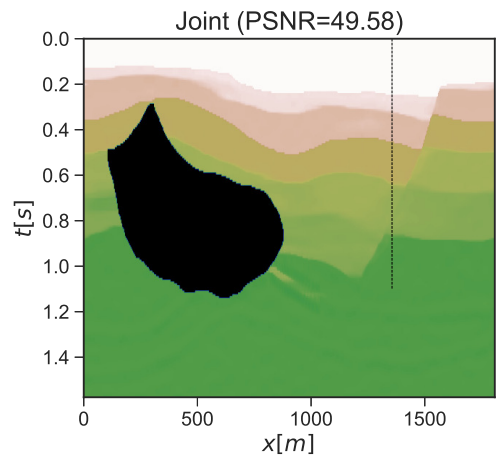

(b)

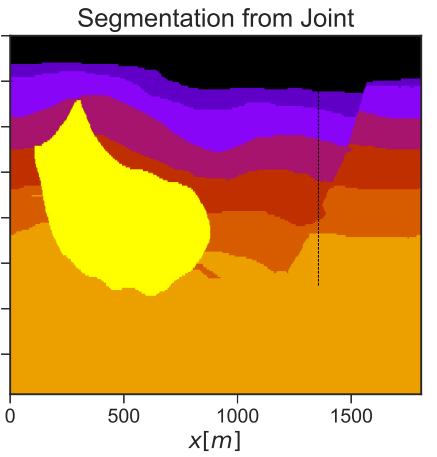

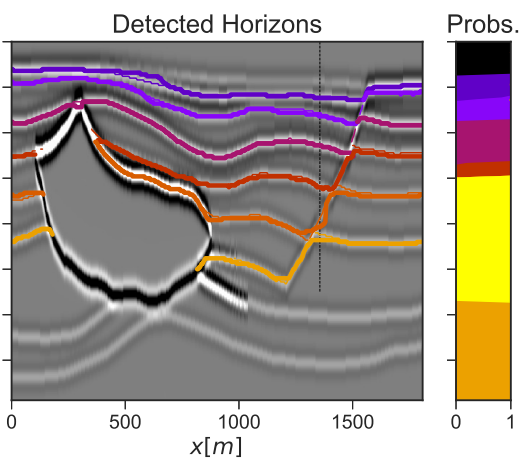

Figure 12. Results for model with short well. a) Inverted model from joint inversion scheme, b) segmentation from the joint inversion scheme, c) tracked horizons overlain on the noise-free seismic dataset, and d) probabilities for each class at reference location. 
To begin with, the acoustic impedance profile is used to identify classes for the segmentation algorithm. Three different zones are chosen, separated by the Shetland and Viking tops, based on the histograms of their acoustic impedance values plotted in Figure 14. However, given the large overlap in the histograms from the overburden class and the Viking class, they are aggregated into a single class. A second class is defined to include most of the values in the Shetland formation and two other classes are defined to capture the low and high acoustic impedance values observed in the well log. Ultimately, 4 different classes $(\mathbf{c}=[4000,7600,10200,13000])$ are used as input to our segmentation algorithm. Note that our ability to successfully separate different formations is highly dependant on the choice of these classes. To accurately fine tune such a selection, it may be useful to perform a first independent step of inversion and apply a pixel-wise segmentation based on the closeness of each pixel value to the classes values. This is equivalent to solving the segmentation problem in step 3 of the Algorithm without the TV-regularization term $(\beta=0)$ leading to a very noisy segmentation result. Nevertheless, by closely inspecting this segmentation result, the classes boundaries can be further optimized prior to running the entire joint inversion-segmentation scheme. We elaborate on this further in the Discussion section.

To be able to invert the seismic data for an acoustic impedance model, a background model is built from the root-mean-square (RMS) velocity model. RMS velocities are first converted into interval velocities and subsequently calibrated with the acoustic impedance log of the NO/15-9 19 BT2 well. More specifically, the interval velocity model is extracted along the well trajectory and used to 'predict' a smoothed version of the acoustic log: prediction is achieved via linear regression and the regression coefficients are further used to convert the entire velocity model into a background acoustic impedance model (Figure 15a). Note that other approaches to construct the low-frequency model can be equivalently used in cases where a larger coverage of vertical wells with the required set of $\operatorname{logs}$ is available. Estimated acoustic impedance models for three different optimization problems are shown in the other panels of Figure 15: spatially regularized least-squares inversion, TV regularized inversion, and our joint inversion-segmentation. As observed in the other examples, TV regularized inversion improves on the blockiness of the model compared to least-squares inversion and produces sharper transitions between different formations; this is especially the case for areas with low and high acoustic impedance values which are generally under predicted due to the lack of low frequency information in the data (see close-ups in Figure 16). After two outer iterations of the Algorithm, this behaviour is even more visible as a consequence of the second regularization term in equation 1 , which drives acoustic impedance values closer to that of the class to which they belong. Moreover, whilst the other two inversion results show some vertical striping in an area of poorer data quality (white arrows), the joint inversion manages to improve the lateral continuity of the model without compromising on 
the sharpness of the vertical transitions. Finally, an overall good match is observed with the acoustic impedance log along the well trajectory as shown in Figure 15e.

Figure 17 displays the segmented model at the end of the joint inversion scheme alongside the probabilities of each class along the well trajectory. White lines represent the different horizons that have been extracted from the different class probabilities. Due to the complexity of the model and the fact that some pairs of classes repeat at different depths, the horizon tracking algorithm is run in this case only considering the class above during the labelling and combination steps. The tracked horizons are also shown in Figure 17c alongside the manually interpreted horizons (thin black lines) on top of the input seismic data. A number of interesting observations can be made with respect to the ability of our algorithm to interpret the key horizons in this data: first, the Top Shetland formation is successfully tracked and the resulting horizon is very similar to the manually interpreted one. On the other hand, our interpretation of Top Ty diverges from the manual interpretation towards the right síde of the model; a similar behaviour is also observed for the horizon above (red line in Figure 17c). By looking at the inverted acoustic impedance (Figure 18b), we can observe a thinning of the Ty formation which is consistent with the tracked horizons. Whilst the quality of the seismic data in this area does not allow us to determine whether our interpreted horizons are correct, this result highlights the direct connection between the tracking algorithm and the inversion step. Future work will investigate this behaviour by both inverting the entire $3 \mathrm{~d}$ dataset and looking at its consistency in the perpendicular direction as well as looking at the pre-stack data in this area. Finally, our algorithm is only able to track part of the BCU horizon, failing to do so when the thin low acoustic impedance layer is eroded out on the right side of the model. Overall, the results in this section confirm the validity of our algorithm and its usefulness in jointly solving a number of interpretation tasks and keeping consistency among the different results: moreover, whilst the acoustic impedance model alone may be useful to condition facies and property modelling, the segmentation model may complement it in defining different areas of influence which may be conditioned differently.

\section{DISCUSSION}

We have presented a novel inversion algorithm for seismic post-stack data that can jointly produce an acoustic impedance model of Earth and a segmented representation of the subsurface in terms of discrete rock units, where the acoustic impedance of each rock unit can be chosen a priori based on our knowledge of the geological setting. We believe this framework to be very suitable for mature fields with extensive amounts of well data and firm geological understanding. On the other hand, we argue that it is less appropriate within an exploration setup where lack of well data and preliminary geological understanding may bias the solution of the inversion towards a very uncertain prior knowledge. 

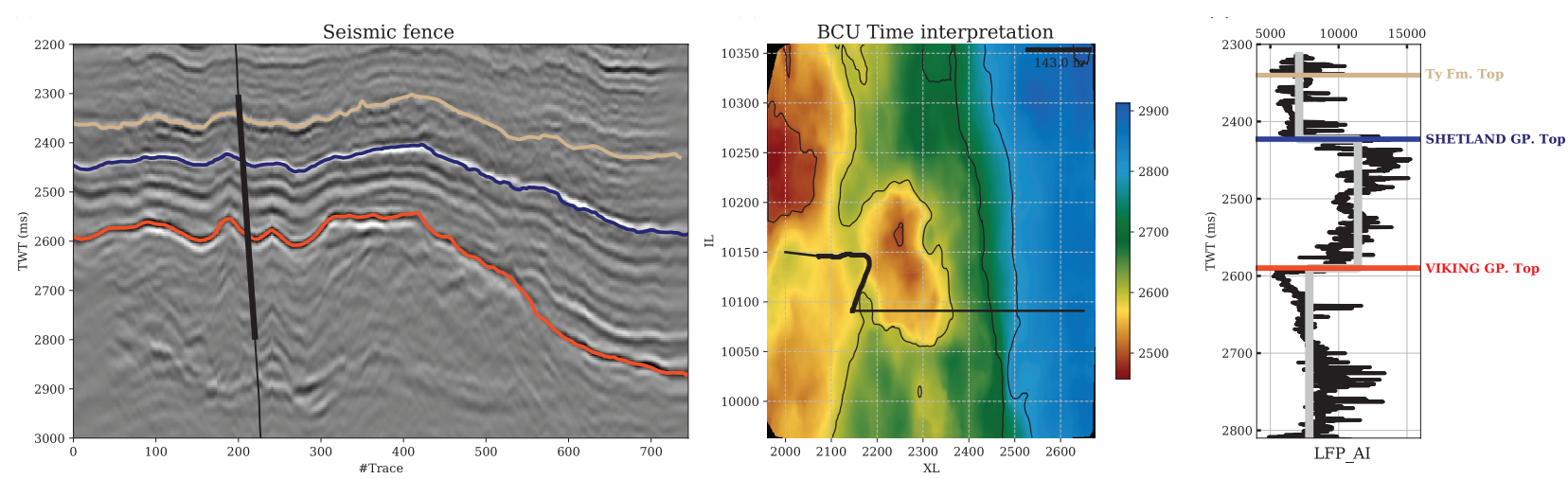

Figure 13. a) Seismic section along the NO/15-9 19 BT2 well with Top Ty (brown line), Top Shetland (blue line) and BCU (red line) horizons. b) BCU time horizon with well trajectory and fence along which seismic is extracted and displayed in the previous panel. c) Acoustic impedance well log and well markers for the 3 formations of interest. All data displayed here is taken from the official Volve dataset.

Apart from the input seismic data and a background impedance model, the outcomes of our algorithm depend on five other input parameters: the number of classes $N_{c}$ and their center values $\mathbf{c}$, and the three tuning parameters $\alpha, \beta$, and $\delta$ associated with the different regularizers of the cost function in equation 1. As generally done for inversion algorithms of such a kind, the parameters $\alpha$ and $\beta$ associated with the two TV regularization terms are manually-tuned based on how blocky the user wishes the acoustic impedance and segmentation model to be, respectively. Based on our numerical results, we argue that whilst adding blockiness constraints comes at the added cost of having to solve multiple nested optimization problems by means of proximal algorithms (e.g., Primal-Dual), this provides a great uplift in the estimated model paramaters when compared to more conventional least-squares

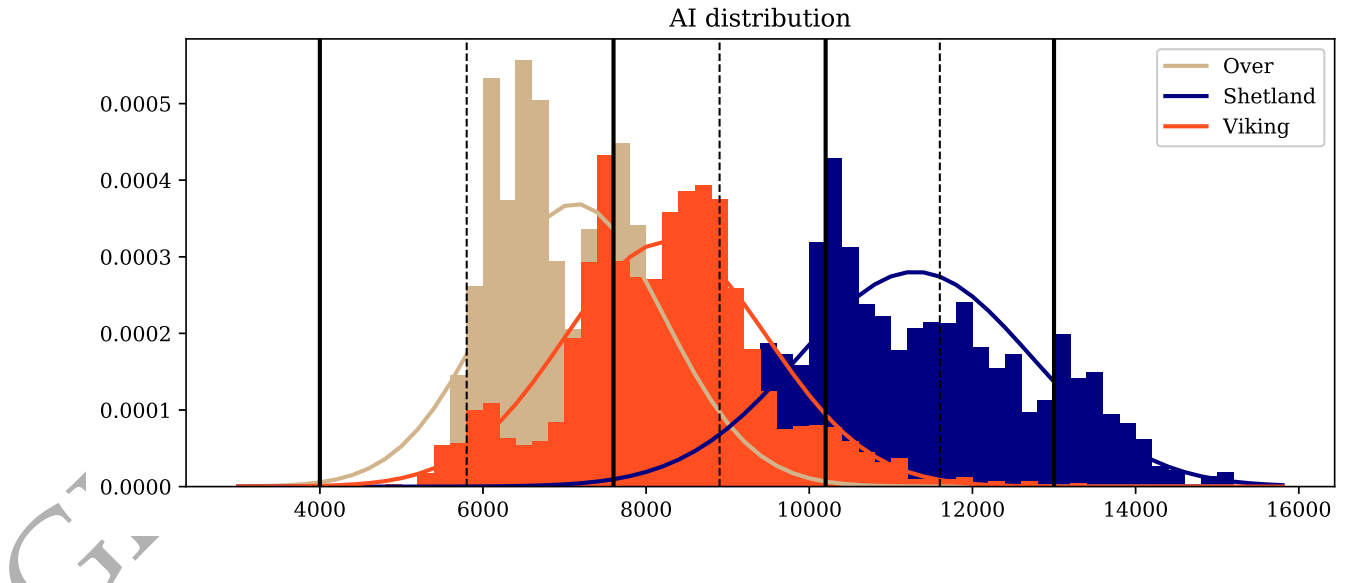

Figure 14. Histogram of the acoustic impedance values at NO/15-9 19 BT2 well divided in zones as defined in Figure 13c. Solid black vertical lines refer to the classes chosen for the segmentation algorithm whilst dashed black vertical lines represent the separation between the classes. 
(a)

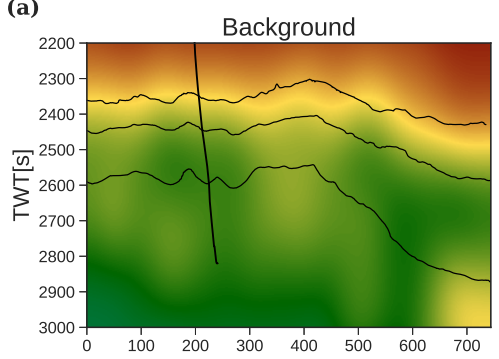

(c)

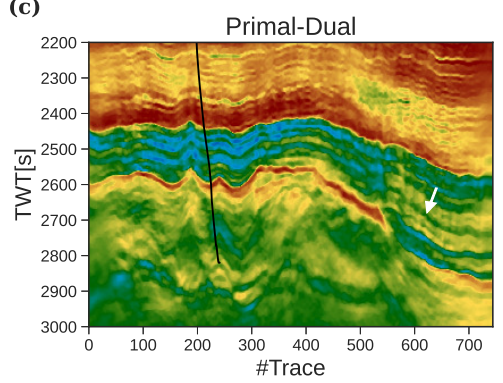

(b)

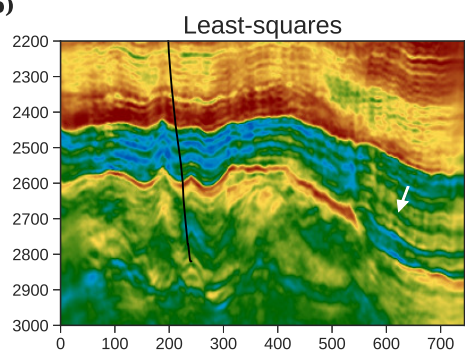

(d)

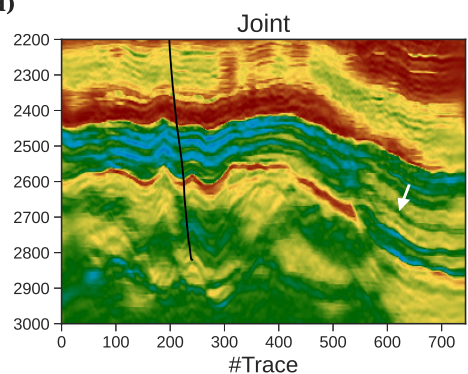

(e)

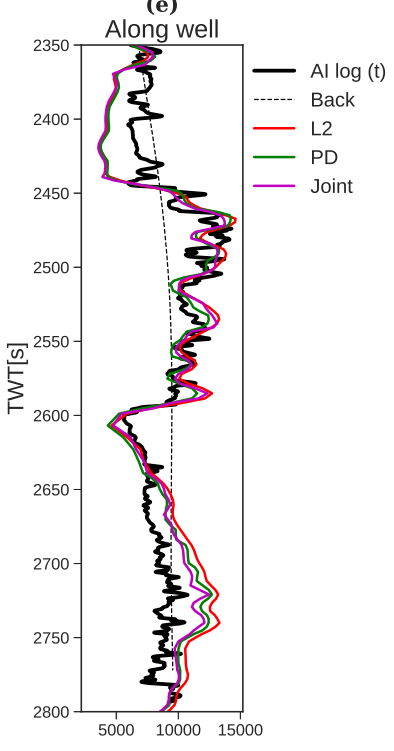

Figure 16. Zoomed sections of acoustic impedance inversion for a) least-squares solver, b) Primal-dual solver and c) Joint inversion. 

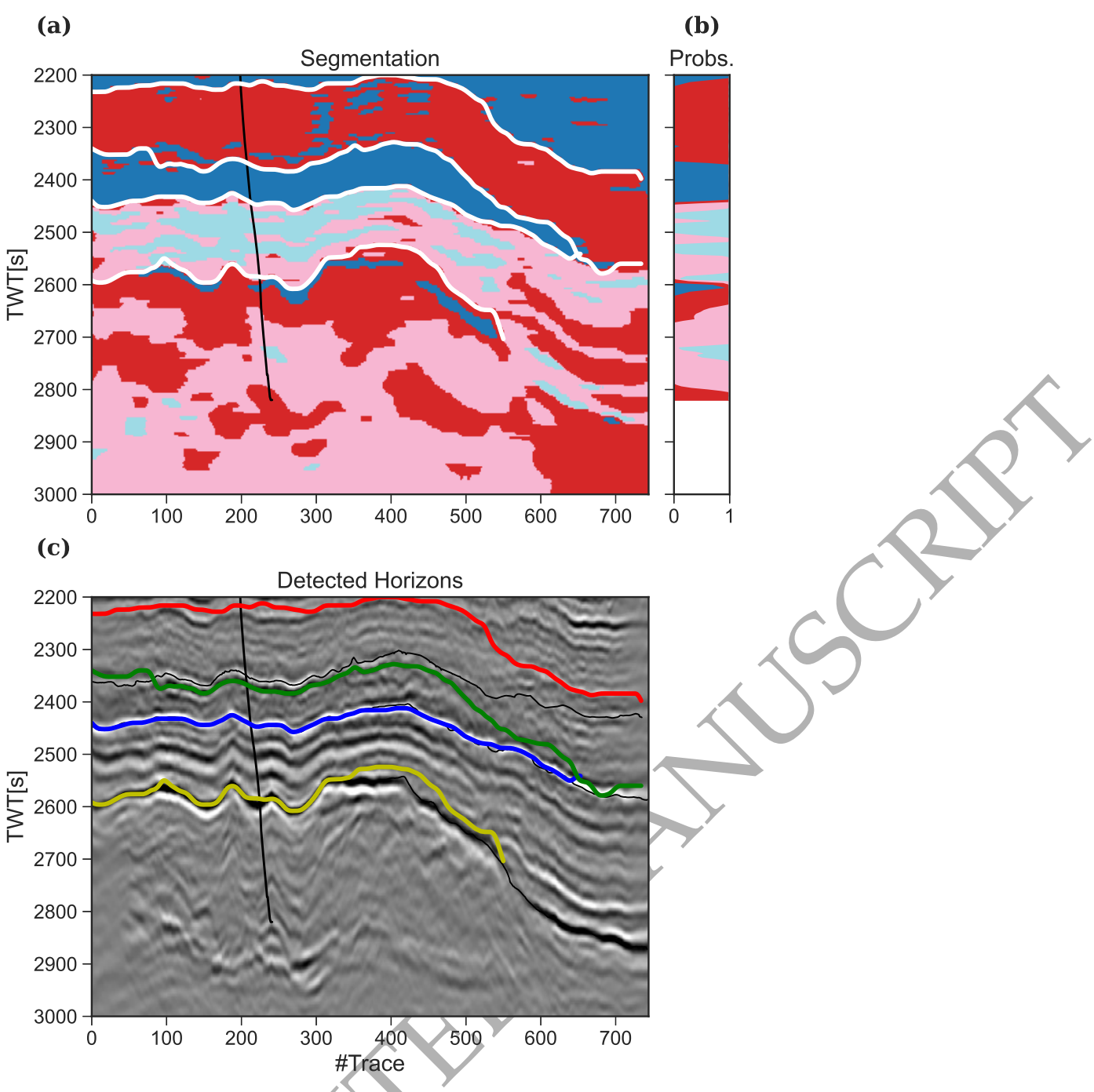

Figure 17. a) Segmentation model with tracked horizons (white lines), b) probabilities for each class at well location, and c) seismic data with tracked horizons.

first on the parameter $\delta$, Figure 19 shows the estimated acoustic impedance model for the Volve dataset when we use a much larger value for $\delta$ than the one selected in our main experiment. As a result of giving a strong weight to the segmentation term (equation 4) in the overall inverse process, the retrieved acoustic impedance model becomes very similar to the segmentation model as it is mostly dominated by the values of the $\mathbf{c}$ vector. Considering the definition of discrete rock classes, we decide to compare three different choices for the Volve example (Figure 20): i) use of 3 classes; ii) use of 4 classes with slightly different centroids than those used in the main example; iii) use of 5 classes. As explained in the Volve dataset section, we begin by applying a first step of inversion and perform pixel-wise segmentation for these three different selections of $N_{c}$ and $\mathbf{c}$ (top row of Figure 20). The corresponding final segmentation results are shown in the bottom row of Figure 20. We observe that 
(a)

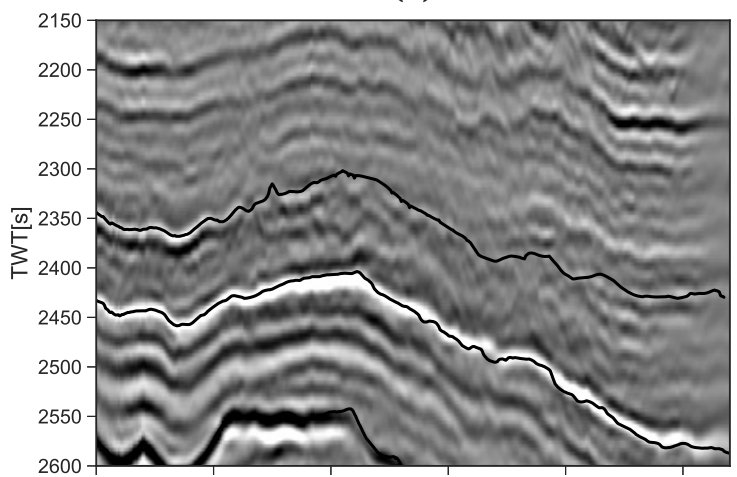

(c)

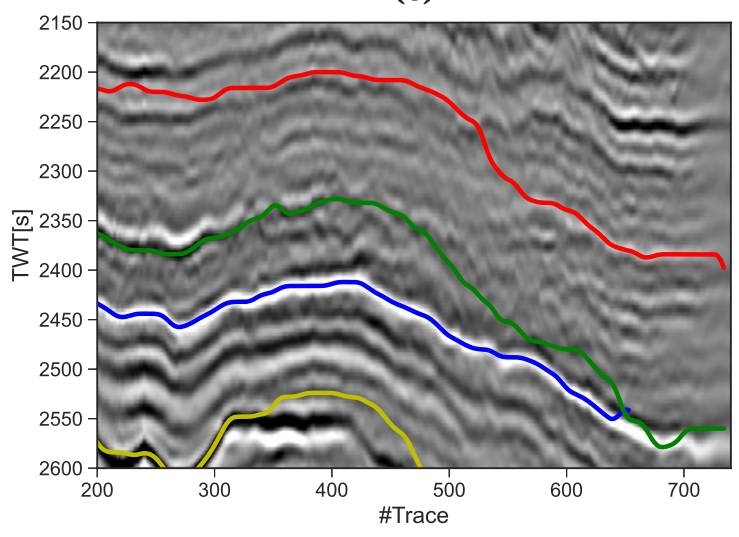

(b)

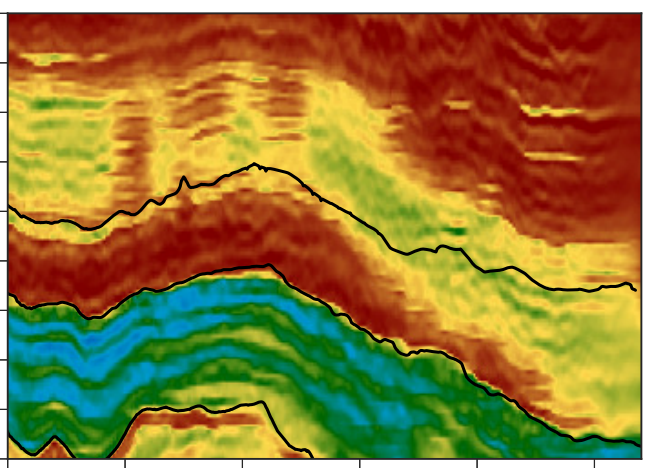

(d)

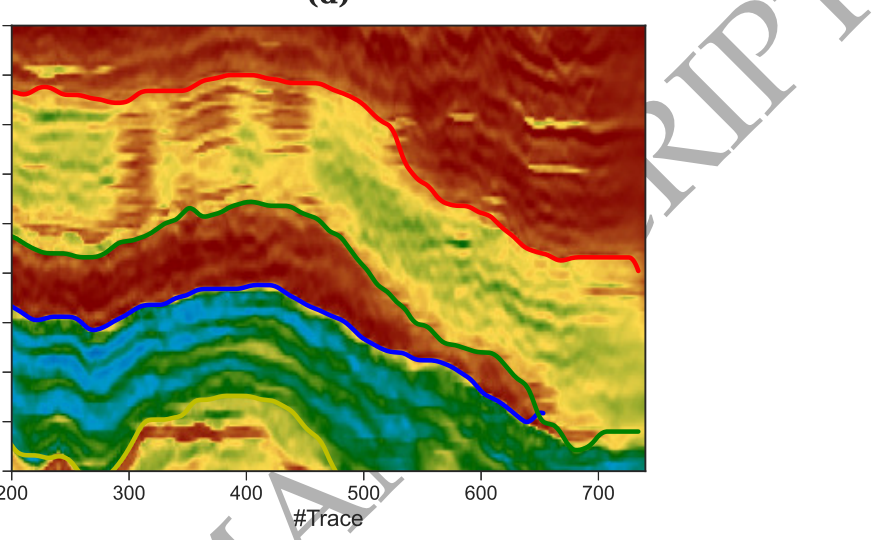

Figure 18. Zoomed sections of a-c) seismic data and b-d) acoustic impedance inversion with interpreted horizons (top: original interpretation, bottom: our interpretation).

the main geological units are successfully recovered for the first two cases, with the main difference that some of the small geobodies with high AI (light blue in Figure 20b) are incorporated in the red class in Figure 20a. On the other hand, the result in Figure 20c reveals an over-parametrisation of the geological units, which ultimately results in very poor identification of the main geological boundaries (white lines in Figure 20f). Focusing on the bottom row, it is possible to appreciate the effect of the $\mathrm{TV}$ regularization term as well as that of running a number of outer iteration on the segmented model, where small-scale features disappear and more continuous geological units are generated.

Finally, the proposed workflow open doors to a number of opportunities for future research. First, the estimated class probabilities (rows of $\mathbf{V}$ ) could be further exploited to provide an uncertainty estimation of the tracked horizons. An initial attempt for the Hess and Volve datasets is reported in Figure 21, where the TV norm of every class probability field are computed and summed together (herein referred to as TV-uncertainty). By exploiting the fact that a sharp transition between two class probabilities corresponds to a thin line in the TV-uncertainty, whilst more uncertain transitions lead to thicker lines in the TV-uncertainty, horizon uncertainties are defined based on the thickness of these lines in correspondence of the tracked horizons. Whilst this strategy can directly leverage our seg- 


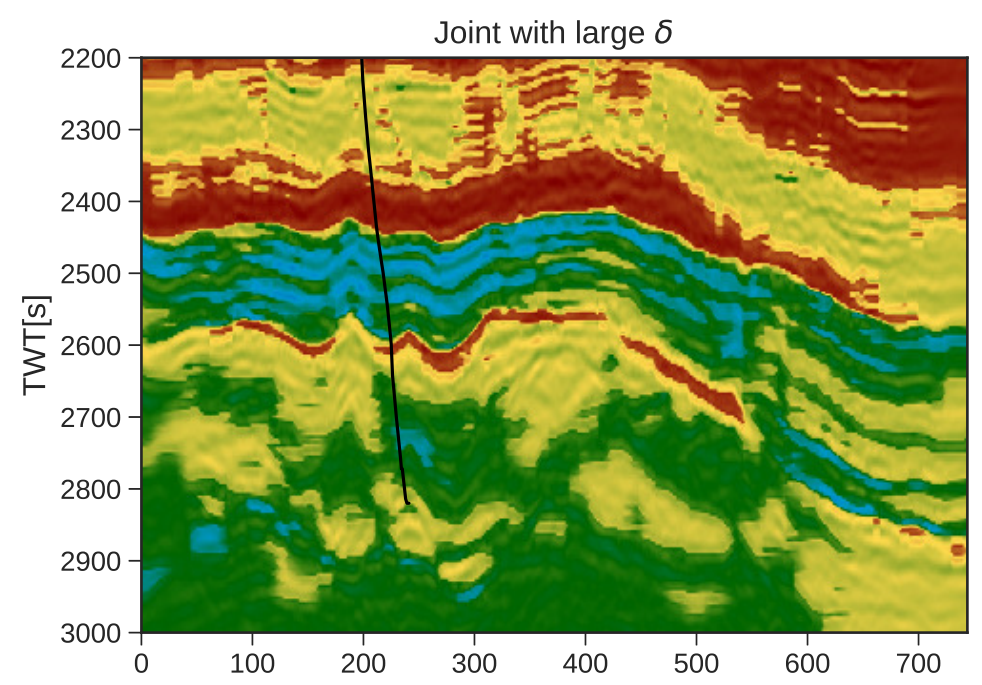

Figure 19. Acoustic impedance model estimated sing a large $\delta$ value.

mentation to produce a relative estimate of the horizon uncertainties, future research will investigate how to transform this into an absolute error bound with the help of well controls. Second, our algorithm presented can be easily adapted to the case of pre-stack seismic data with the possibly added benefit of better separation between the different classes chosen as input to the segmentation step. As
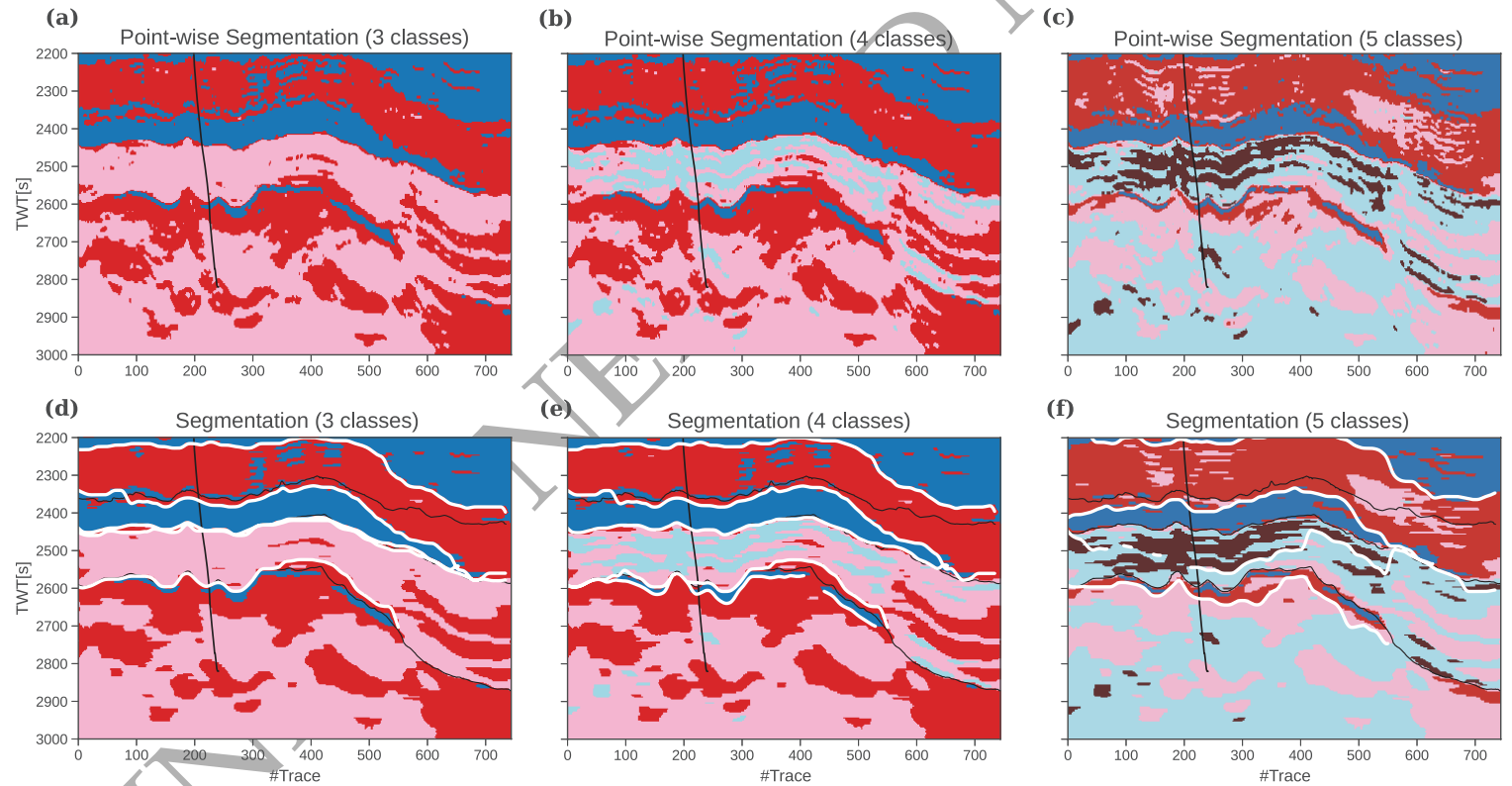

(e) Segmentation (4 classes)

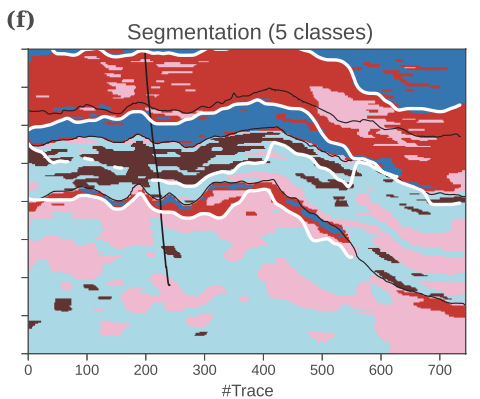

Figure 20. Sensitivity analysis on the selection of the number of classes $N_{c}$ and class vector c. Point-wise segmentation (top) and segmentation from joint inversion-segmentation algorithm (bottom) for a-d) 3 classes with $\mathbf{c}=[4000,7600,10200]$, b-e) 4 classes with $\mathbf{c}=[3950,7640,10140,12970]$, c-f) 5 classes with $\mathbf{c}=$ [4000, 6000, 7600, 10200, 13000]. 
far as the inversion step is concerned, the model vector $\mathbf{m}$ can simply be defined as a vector of size $N_{m} N_{x} N_{z} \times 1$ where $N_{m}$ is the number of model parameters we wish to invert for (generally 2 or 3 ). Similarly, we can define the data vector $\mathbf{d}$ as a vector of size $N_{\theta} N_{x} N_{z} \times 1$ where $N_{\theta}$ is the number of angles. Finally, the modelling operator is redefined as $\mathbf{G}=\mathbf{W R D}$ to include a mixing matrix $\mathbf{R}$ which contains the weighting coefficients of the different elastic reflectivities used to model the amplitude variation with offset (AVO) seismic response - e.g., Aki-Richards. Similar to the matrix V, we can also consider a matrix $\mathbf{M}$ of size $N_{x} N_{z} \times N_{m}$ (where $\mathbf{m}=V e c(\mathbf{M})$ is its vectorized version obtained by concatenating the matrix's columns) and replace the TV regularization term in equation 1 by the sum of $N_{m}$ TV norms acting on each column of $\mathbf{M}$. The proximal operator of this term can therefore be computed in the same way as that of $\mathbf{V}$ (equation A.2). Alternatively, as described in Causse et al. (2007) and Ravasi et al. (2017), the different model parameters can be first decoupled from each other, followed by $N_{m}$ independent steps of inversion that are identical to the one employed in the post-stack scenario. Similarly, for the segmentation step, we can define a matrix $\mathbf{C}$ of size $N_{c} \times N_{m}$ whose rows contain the set of elastic parameters associated to each class and replace the $\left(m_{i}-c_{j}\right)$ in equation 1 by the Euclidean norm of the difference between each row of $\mathbf{M}$ and $\mathbf{C}$ (i.e., $\left\|\mathbf{M}_{i}^{T}-\mathbf{C}_{j}^{T}\right\|_{2}$ ).

\section{CONCLUSIONS}

In this work we have presented an end-to-end approach for assisted seismic interpretation, which provides, as well as a set of interpreted horizons, a property model and a segmented model of the subsurface. Acoustic (or elastic) properties are not only inverted for the entire model from seismic data, but they are also used as input to define an easy to understand link between well log responses and the expected horizons to be extracted in the final step of our workflow. This in turn reconciles classical principles of seismic interpretation to the proposed optimization problem, making the method easier to explain to and be used by skilled interpreters. Our work remarks on the importance of enforcing blockiness in the inversion of seismic data, which is obtained via a combination of regularization (TV regularization), solver selection (Primal-Dual) and additional constraints (segmentation model): our estimates present sharper jumps at model discontinuities than their least-squares counterpart and use prior knowledge to compensate for the lack of low and high frequencies in seismic data. The ability to retrieve more broadband models not only helps the subsequent segmentation step, but it can also have strong implications when such models are used as hard or soft constraints in downstream processes such as facies or property modelling. Finally, whilst an extension of our inversion algorithm to pre-stack seismic data has been discussed, we foresee its applicability to other linear (and nonlinear) inverse problems in geophysics, such as least-squares migration and waveform inversion. 
(a)

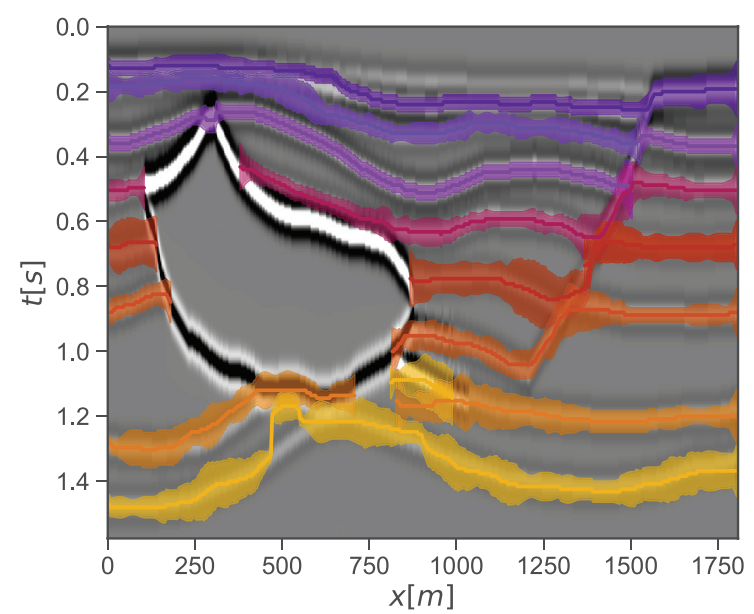

(b)

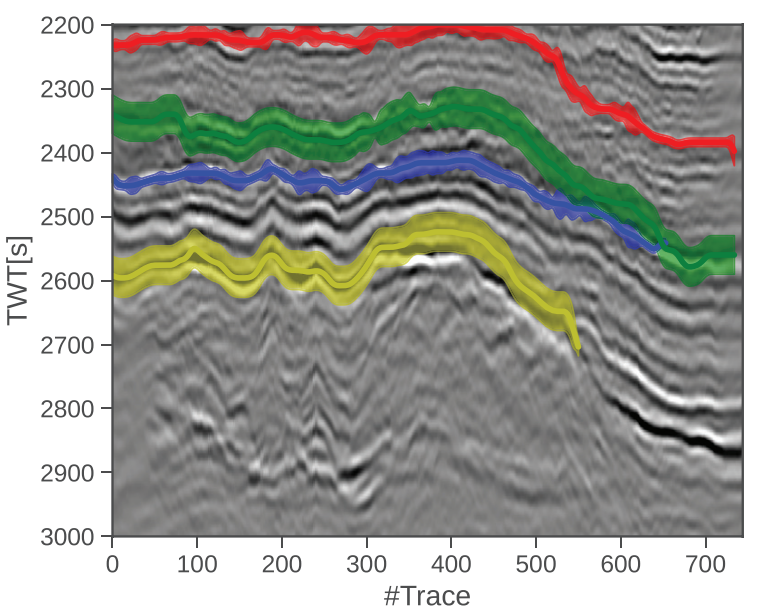

Figure 21. Horizon uncertainties for a) Hess dataset and b) Volve dataset.

\section{DATA AVAILABILITY}

The code and data underlying this article are available under permissive licence at https://github.com/DIGKaust/HTracker .

\section{REFERENCES}

Angelo, M. V. D., Fifariz, R., Meckel, T., \& Trevino, R. H,, 2019. A seismic-based CO2-sequestration regional assessment of the miocene section, northern gulf of mexico, texas and louisiana, International Journal of Greenhouse Gas Control, pp. 29-37.

Ashcroft, W., 2011. A Petroleum Geologist's Guide to Seismic Reflection, Wiley.

Benning, M. \& Burger, M., 2013. Ground states and singular vectors of convex variational regularization methods, Methods Appl Anal., 20, 295-334.

Boyd, S., Parikh, N., Chu, E., Péleato, B., \& Eckstein, J., 2011. Distributed optimization and statistical learning via the alternating direction method of multipliers, Foundations and Trends in Machine Learning, 3(1), $1-122$.

Bredesen, K., Rasmussen, R., Mathiesen, A., \& Nielsen, L., 2021. Seismic amplitude analysis and rock physics modeling of a geothermal sandstone reservoir in the southern part of the danish basin, Geothermics, $\mathbf{8 9}$, doi: 10.1016/j.geothermics.2020.101974.

Bregman, L. M., 1967. The relaxation method of finding the common points of convex sets and its application to the solution of problems in convex programming, USSR Computational Mathematics and Mathematical Physics, 7, 200-217, doi: 10.1016/0041-5553(67)90040-7.

Brown, A., 2011. Interpretation of Three-Dimensional Seismic Data, American Association of Petroleum Geologists. 
Buland, A. \& Omre, H., 2003. Bayesian linearized avo inversion, Geophysics, 68, 185-198, doi: 10.1190/1. 1543206.

Causse, E., Riede, M., Wijngaarden, A. V., Buland, A., Dutzer, J., \& Fillon, R., 2007. Amplitude analysis with an optimal model-based linear avo approximation: Part i-theory, Geophysics, 72(3), C59-C69.

Chambolle, A. \& Pock, T., 2011. A first-order primal-dual algorithm for convex problems with applications to imaging, Journal of Mathematical Imaging and Vision, 40, 120-145.

Chambolle, A., Cremers, D., \& Pock, T., 2012. A convex approach to minimal partitions, SIAM Journal on Imaging Sciences, 5, 1113-1158, doi: 10.1137/110856733.

Cooke, D. A. \& Schneider, W. A., 1983. Generalized linear inversion of reflection seismic data, Geophysics, 48, 665-676, doi: 10.1190/1.1441497.

Corona, V., Benning, M., M. Ehrhardt and., L. G., Mair, R., Reci, A., Sederman, A., Reichelt, S., \& Schönlieb, C.-B., 2019. Enhancing joint reconstruction and segmentation with non-convex bregman iterationt, Inverse Problems.

Gholami, A., 2015. Nonlinear multichannel impedance inversion by total-variation regularization, Geophysics, 80, R217-R224.

Goldstein, T. \& Osher, S., 2009. The split bregman method for 11-regularized problems, SIAM Journal on Scientific Computing, 2(2), 323-343.

Haibin, D., Truelove, L., Li, C., \& Abubakar, A., 2019. Accelerating seismic fault and stratigraphy interpretation with deep cnns: A case study of the taranaki basin, The Leading Edge, 39(10).

Hampson, D., Russell, B., \& Bankhead, B., 2005. Simultaneous inversion of pre-stack seismic data, SEG Technical Program Expanded Abstracts, pp. 1633-1637.

Herron, D. A., 2011. Seismic Intepretation, Society of Exploration Geophysicists.

Kieu, D. T. \& Kepic, A., 2020. Seismic-impedance inversion with fuzzy clustering constraints: an example from the carlin gold district, nevada, usa, Geophysical Prospecting, 68, 103-128, doi: 10.1111/1365-2478. 12891.

Kolbjornsen, O., Evensen, O., Nilsen, A. K., \& Lie, J. E., 2019. Digital superresolution in seismic avo inversion, The Leading Edge, 39, 791-799.

Majer, E., 2003. 3-d seismic methods for geothermal reservoir exploration and assessment, Lawrence Berkeley National Laboratory.

Margrave, G. F., Stewárt, R. R., \& Larsen, J. A., 2001. Joint pp and ps seismic inversion, The Leading Edge, 20, 1048-1052, doi: 10.1190/1.1487311.

Mulrooney, M. Osmond, J., Skurtveit, E., Wu, L., \& Braathen, A., 2018. Smeaheia, a potential northern north sea $\mathrm{CO} 2$ storage site: Structural description and de-risking strategies, Fifth CO2 Geological Storage Workshop, European Association of Geoscientists amp; Engineers, pp. 1-5.

Oldenburg, D. W., Scheuer, T., \& Levy, S., 1983. Recovery of the acoustic impedance from reflection seismograms, Geophysics, 48, 1318-1337, doi: 10.1190/1.1441413.

Osher, S., Burger, M., Goldfarb, G., Xu, J., \& Yin, W., 2005. An iterative regularization method for total 
variation-based image restoration, Multiscale Model. Simulation, 4, 460-489.

Paige, C. C. \& Saunders, M. A., 1982. Lsqr: An algorithm for sparse linear equations and sparse least squares, TOMS, 8, 43-71.

Parikh, N., 2013. Proximal Algorithms, Foundations and Trends in Optimization.

Qiu, L., Chemingui, N., Zou, Z., \& Valenciano, A., 2016. Full-waveform inversion with steerable variation regularization, in SEG Technical Program Expanded Abstracts, pp. 1174-1178, doi: 10.1190/ segam2016-13872436.1.

Ravasi, M. \& Vasconcelos, I., 2020. Pylops - a linear-operator python library for scalable algebra and optimization, SoftwareX, 11, 100361.

Ravasi, M., Alerini, M., Maultzsch, S., \& Ghaderi, A., 2017. Band-limited optavo, seismic inversion the other way round, 79th EAGE Conference and Exhibition.

Russell, B. \& Hampson, D., 2011. A comparison of post-stack seismic inversion methods, in SEG Technical Program Expanded Abstracts, pp. 876-878, doi: 10.1190/1.1888870.

Sheriff, R. E., 2002. Encyclopedic dictionary of applied geophysics, Society of Exploration Geophysicists.

Shi, Y., Wu, X., \& Fomel, S., 2019. Saltseg: Automatic 3d salt segmentation using a deep convolutional neural network, Interpretation, 7(3), SE113-SE1221.

Shi, Y., Wu, X., \& Fomel, S., 2020. Waveform embedding: Automatic horizon picking with unsupervised deep learning, Interpretation, 85(4), 1-48.

Simmons, J. L. \& Backus, M. M., 1996. Waveform-based avo inversion and avo prediction-error, Geophysics, 61, 1575-1588, doi: 10.1190/1.1444077.

Sun, J. \& Li, Y., 2015. Multidomain petrophysically constrained inversion and geology differentiation using guided fuzzy c-means clustering, Geophysics, 80, ID1-ID18, doi: 10.1190/geo2014-0049.1.

Sun, S., Yin, C., \& Gao, X., 2001. 3d gravity inversion on unstructured grids, Applied Sciences, 2, 1048-1052, doi: $10.3390 /$ app11020722.

Waldeland, A. U., Jensen, A. C., Gelius, L.-J., \& Solberg, A., 2018. Convolutional neural networks for automated seismic interpretation, The Leading Edge, 37(4), 529-537.

Wang, D., Gao, J., \& Zhou, H., 2019. Data-driven multichannel seismic impedance inversion with anisotropic total variation regularization, Journal of Inverse and Ill-Posed Problems, 2018(2), 229-241.

\section{Appendix A: Implementation of proximal operators}

This Appendix describes each of the proximal operators appearing in the Algorithm and details their numerical implementation. To begin with, the proximal operator of $g=\frac{1}{2}\|\hat{\mathbf{d}}-\hat{\mathbf{G}} \mathbf{m}\|_{2}^{2}$ in step 1 of the Algorithm is that of a quadratic functional which can be written as:

$$
\operatorname{prox}_{\tau g}(\mathbf{m})=\left(\mathbf{I}+\tau \hat{\mathbf{G}}^{T} \hat{\mathbf{G}}\right)^{-1}\left(\mathbf{m}+\tau \hat{\mathbf{G}}^{T} \hat{\mathbf{d}}\right)
$$

The evaluation of such a proximal operator requires the solution of a regularized least-squares 
inverse problem. Apart from some specific cases involving orthogonal operators, where an analytical solution can be identified (e.g., CT scan reconstruction - Corona et al. (2019)), the evaluation of such an operator is generally the most expensive of the operators involved in our algorithm and requires the use of iterative solvers (e.g., CGLS, LSQR). However, as each iteration of the Primal-Dual algorithm represents only a step towards the saddle point of the corresponding Primal-Dual functional, an approximated solution of the problem obtained with an early stopping after a limited number of iterations generally suffices. Moreover, a warm start is generally employed, meaning that the solution of the proximal operator from its previous evaluation is used as starting guess of its current evaluation. This often provides a very large speed improvement over solving the problem from scratch each time (Parikh 2013).

The proximal operator of $f=\|\|_{2,1}$ in steps 1 and 3 of the Algorithm is that of the $L_{2,1}$ norm i.e. sum of the Euclidean norms of the columns of a matrix $\mathbf{Y}$ - which is:

$$
\operatorname{prox}_{\tau f}(\mathbf{Y})=\left(\operatorname{prox}_{\tau f}\left(\mathbf{Y}_{0}\right)^{T}, \ldots, \operatorname{prox}_{\tau f}\left(\mathbf{Y}_{N}\right)^{T}\right)^{T}, \quad \operatorname{prox}_{\tau f}(\mathbf{y})=\left(1-\frac{\sigma \tau}{\max \left\{\|\mathbf{y}\|_{2}, \tau\right\}}\right) \mathbf{y}
$$

As show in equation A.2, such an operator requires only point-wise evaluations for each element of the input vector; this is therefore very fast to compute, even for very large two or three dimensional models.

Finally, the proximal operator of the unit Simplex used within the segmentation process (function $g$ in step 3 of the Algorithm) also requires only point-wise evaluations, where each column of the matrix $\mathbf{V}$ is treated independently and projected over the intersection of a hyperplane and a box. This is implemented as the evaluation of a projection over a box:

$$
P_{C}=P_{B o x} Y_{[0,+\infty]}\left(\mathbf{x}-\mu^{*} \mathbf{1}\right)
$$

where $\mu^{*}$ is the solution of the following scalar equation

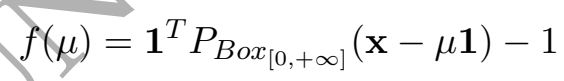

which can be solved via bisection. The cost of this operation is dependant on the speed of convergence of the bisection algorithm and may require tens of evaluations of the function $f$. Note that, although this has a higher cost compared to the proximal operator of the $L_{2,1}$ norm, its implementation can be made very fast when implemented on modern, massively parallel hardware (i.e., GPUs). 